\title{
On the augmentation quotients of the IA-automorphism group of a free group
}

\author{
TAKAO SATOH
}

\begin{abstract}
We study the augmentation quotients of the IA-automorphism group of a free group and a free metabelian group. First, for any group $G$, we construct a lift of the $k-$ th Johnson homomorphism of the automorphism group of $G$ to the $k$-th augmentation quotient of the IA-automorphism group of $G$. Then we study the images of these homomorphisms for the case where $G$ is a free group and a free metabelian group. As a corollary, we detect a $\mathbb{Z}$-free part in each of the augmentation quotients, which can not be detected by the abelianization of the IA-automorphism group.
\end{abstract}

20F28; 16 S34

\section{Introduction}

Let $F_{n}$ be a free group of rank $n \geq 2$, and Aut $F_{n}$ the automorphism group of $F_{n}$. Let $\rho:$ Aut $F_{n} \rightarrow$ Aut $H$ denote the natural homomorphism induced from the abelianization $F_{n} \rightarrow H$. The kernel of $\rho$ is called the IA-automorphism group of $F_{n}$, denoted by $\mathrm{IA}_{n}$. The subgroup $\mathrm{IA}_{n}$ reflects much of the richness and complexity of the structure of Aut $F_{n}$ and plays important roles in various studies of Aut $F_{n}$. Although the study of the IA-automorphism group has a long history since its finitely many generators were obtained by Magnus [13] in 1935, the combinatorial group structure of $\mathrm{IA}_{n}$ is still quite complicated. For instance, no presentation for $\mathrm{IA}_{n}$ is known in general.

We have studied $\mathrm{IA}_{n}$ mainly using the Johnson filtration of Aut $F_{n}$ so far. The Johnson filtration is one of a descending central series

$$
\mathrm{IA}_{n}=\mathcal{A}_{n}(1) \supset \mathcal{A}_{n}(2) \supset \cdots
$$

consisting of normal subgroups of Aut $F_{n}$, whose first term is $\mathrm{IA}_{n}$. (For details, see Section 2.3.) Each graded quotient $\operatorname{gr}^{k}\left(\mathcal{A}_{n}\right):=\mathcal{A}_{n}(k) / \mathcal{A}_{n}(k+1)$ naturally has a $\mathrm{GL}(n, \mathbf{Z})$-module structure, and from it we can extract some valuable information about $\mathrm{IA}_{n}$. For example, $\operatorname{gr}^{1}\left(\mathcal{A}_{n}\right)$ is just the abelianization of $\mathrm{IA}_{n}$ due to Cohen and Pakianathan [6; 7], Farb [8] and Kawazumi [12]. Pettet [18] determined the image of the cup product $\cup_{\mathbf{Q}}: \Lambda^{2} H^{1}\left(\mathrm{IA}_{n}, \mathbf{Q}\right) \rightarrow H^{2}\left(\mathrm{IA}_{n}, \mathbf{Q}\right)$ by using the $\operatorname{GL}(n, \mathbf{Q})$-module 
structure of $\operatorname{gr}^{2}\left(\mathcal{A}_{n}\right) \otimes \mathbf{Z} \mathbf{Q} s$. At the present stage, however, the structures of the graded quotients $\operatorname{gr}^{k}\left(\mathcal{A}_{n}\right)$ are far from well-known.

On the other hand, compared with the Johnson filtration, the lower central series $\Gamma_{\mathrm{IA}_{n}}(k)$ of $\mathrm{IA}_{n}$ and its graded quotients $\mathcal{L}_{\mathrm{IA}_{n}}(k):=\Gamma_{\mathrm{IA}_{n}}(k) / \Gamma_{\mathrm{IA}_{n}}(k+1)$ are somewhat easier to handle since we can obtain finitely many generators of $\mathcal{L}_{\mathrm{IA}_{n}}(k)$ using the Magnus generators of $\mathrm{IA}_{n}$. Since the Johnson filtration is central, $\Gamma_{\mathrm{IA}_{n}}(k) \subset \mathcal{A}_{n}(k)$ for any $k \geq 1$. Andreadakis conjectured that $\Gamma_{\mathrm{IA}_{n}}(k)=\mathcal{A}_{n}(k)$ for each $k \geq 1$ and showed $\Gamma_{\mathrm{IA}_{2}}(k)=\mathcal{A}_{2}(k)$ for each $k \geq 1$. It is currently known that $\Gamma_{\mathrm{IA}_{n}}(2)=\mathcal{A}_{n}(2)$ due to Bachmuth [2], and that $\Gamma_{\mathrm{IA}_{n}}(3)$ has at most finite index in $\mathcal{A}_{n}(3)$ due to Pettet [18].

In this paper, we consider the augmentation quotients of $\mathrm{IA}_{n}$. Let $\mathbf{Z}[G]$ be the integral group ring of a group $G$, and $\Delta(G)$ the augmentation ideal of $\mathbf{Z}[G]$. We denote by $Q^{k}(G):=\Delta^{k}(G) / \Delta^{k+1}(G)$ the $k$-th augmentation quotient of $G$. The augmentation quotients $Q^{k}\left(\mathrm{IA}_{n}\right)$ of $\mathrm{IA}_{n}$ seem to be closely related to the lower central series $\Gamma_{\mathrm{IA}_{n}}(k)$ as follows. If Andreadakis' conjecture is true, then each of the graded quotients $\mathcal{L}_{\mathrm{IA}_{n}}(k)$ is free abelian. Using a work of Sandling and Tahara [20] (for details, see Section 4.1), we obtain a conjecture for the $\mathbf{Z}$-module structure of $Q^{k}\left(\mathrm{IA}_{n}\right)$ :

Conjecture 1 For any $k \geq 1$,

$$
Q^{k}\left(\mathrm{IA}_{n}\right) \cong \sum \bigotimes_{i=1}^{k} S^{a_{i}}\left(\mathcal{L}_{\mathrm{IA}_{n}}(i)\right)
$$

as a $\mathbf{Z}$-module. Here the sum runs over all nonnegative integers $a_{1}, \ldots, a_{k}$ such that $\sum_{i=1}^{k} i a_{i}=k$, and $S^{a}(M)$ means the symmetric tensor product of a $\mathbf{Z}$-module $M$ such that $S^{0}(M)=\mathbf{Z}$.

We see that this is true for $k=1$ and 2 from a general argument in group ring theory. (For $k=2$, see (1) below.) For $k \geq 3$, however, it is still an open problem. In general, one of the most standard methods to study the augmentation quotients $Q^{k}\left(\mathrm{IA}_{n}\right)$ is to consider a natural surjective homomorphism $\pi_{k}: Q^{k}\left(\mathrm{IA}_{n}\right) \rightarrow Q^{k}\left(\mathrm{IA}_{n}^{\mathrm{ab}}\right)$ induced from the abelianization $\mathrm{IA}_{n} \rightarrow \mathrm{IA}_{n}^{\mathrm{ab}}$ of $\mathrm{IA}_{n}$. Furthermore, since $\mathrm{IA}_{n}^{\mathrm{ab}}$ is free abelian, we have a natural isomorphism $Q^{k}\left(\mathrm{IA}_{n}^{\mathrm{ab}}\right) \cong S^{k}\left(\mathcal{L}_{\mathrm{IA}_{n}}(1)\right)$. Hence, in the conjecture above, we can detect $S^{k}\left(\mathcal{L}_{\mathrm{IA}_{n}}(1)\right)$ in $Q^{k}\left(\mathrm{IA}_{n}\right)$ by the abelianization of $\mathrm{IA}_{n}$.

Then we have a natural problem to consider: Determine the structure of the kernel of $\pi_{k}$. More precisely, clarify the $\operatorname{GL}(n, \mathbf{Z})$-module structure of $\operatorname{Ker}\left(\pi_{k}\right)$. In order to attack this problem, in this paper we construct and study a certain homomorphism defined on $Q^{k}\left(\mathrm{IA}_{n}\right)$ whose restriction to $\operatorname{Ker}\left(\pi_{k}\right)$ is nontrivial. For a group $G$, let 
$\alpha_{k}=\alpha_{k, G}: \mathcal{L}_{G}(k) \rightarrow Q^{k}(G)$ be a homomorphism defined by $\sigma \mapsto \sigma-1$. One of the main purposes of the paper is to construct a $\operatorname{GL}(n, \mathbf{Z})$-equivariant homomorphism

$$
\mu_{k}: Q^{k}\left(\operatorname{IA}_{n}\right) \rightarrow \operatorname{Hom}_{\mathbf{Z}}\left(H, \alpha_{k+1}\left(\mathcal{L}_{n}(k+1)\right)\right),
$$

where $\mathcal{L}_{n}(k)$ is the $k$-th graded quotient of the lower central series of $F_{n}$. Furthermore, for the $k$-th Johnson homomorphism

$$
\tau_{k}^{\prime}: \mathcal{L}_{\mathrm{IA}_{n}}(k) \rightarrow \operatorname{Hom}_{\mathbf{Z}}\left(H, \mathcal{L}_{n}(k+1)\right)
$$

defined by $\sigma \mapsto\left(x \mapsto x^{-1} x^{\sigma}\right)$ (see Section 2.3 for details), we show that $\mu_{k} \circ \alpha_{k}=$ $\alpha_{k+1}^{*} \circ \tau_{k}^{\prime}$ where $\alpha_{k+1}^{*}$ is a natural homomorphism induced from $\alpha_{k+1}$. Since $\alpha_{k, F_{n}}$ is a $\operatorname{GL}(n, \mathbf{Z})$-equivariant injective homomorphism for each $k \geq 1$, if we identify $\mathcal{L}_{n}(k)$ with its image $\alpha_{k}\left(\mathcal{L}_{n}(k)\right)$, we obtain $\mu_{k} \circ \alpha_{k}=\tau_{k}^{\prime}$. Hence, the homomorphism $\mu_{k}$ can be considered as a lift of the Johnson homomorphism $\tau_{k}^{\prime}$. In the following, we naturally identify $\operatorname{Hom}_{\mathbf{Z}}\left(H, \mathcal{L}_{n}(k+1)\right)$ with $H^{*} \otimes_{\mathbf{Z}} \mathcal{L}_{n}(k+1)$ for $H^{*}:=\operatorname{Hom}_{\mathbf{Z}}(H, \mathbf{Z})$.

Historically, the study of the Johnson homomorphisms was originally begun in 1980 by D Johnson [10] who determined the abelianization of the Torelli subgroup of the mapping class group of a surface in [11]. Now, there is a broad range of remarkable results for the Johnson homomorphisms of the mapping class group. (For example, see Hain [9] and Morita [14; 15; 16].) These works also inspired the study of the Johnson homomorphisms of Aut $F_{n}$. Using it, we can investigate the graded quotients $\operatorname{gr}^{k}\left(\mathcal{A}_{n}\right)$ and $\mathcal{L}_{\mathrm{IA}_{n}}(k)$. Recently, good progress has been achieved by many authors, for example, Cohen and Pakianathan [6; 7], Farb [8], Kawazumi [12], Morita [14; 15; 16] and Pettet [18]. In particular, in our previous work [23], we determined the cokernel of the rational Johnson homomorphism $\tau_{k, \mathbf{Q}}^{\prime}:=\tau_{k}^{\prime} \otimes \operatorname{id}_{\mathbf{Q}}$ for $2 \leq k \leq n-2$.

The main theorem of the paper is:

Theorem 1 (See Theorem 4.4.) For $3 \leq k \leq n-2$, the $\operatorname{GL}(n, \mathbf{Z})$-equivariant homomorphism

$$
\mu_{k} \oplus \pi_{k}: Q^{k}\left(\mathrm{IA}_{n}\right) \rightarrow\left(H^{*} \otimes_{\mathbf{z}} \alpha_{k+1}\left(\mathcal{L}_{n}(k+1)\right)\right) \oplus Q^{k}\left(\mathrm{IA}_{n}^{\mathrm{ab}}\right)
$$

defined by $\sigma \mapsto\left(\mu_{k}(\sigma), \pi_{k}(\sigma)\right)$ is surjective.

Next, we consider the framework above for a free metabelian group. Let $F_{n}^{M}:=$ $F_{n} /\left[\left[F_{n}, F_{n}\right],\left[F_{n}, F_{n}\right]\right]$ be a free metabelian group of rank $n$. By the same argument as the free group case, we can consider the IA-automorphism group $\mathrm{IA}_{n}^{M}$ and the Johnson homomorphism

$$
\tau_{k}^{\prime}: \mathcal{L}_{\mathrm{IA}_{n}^{M}}(k) \rightarrow H^{*} \otimes_{\mathbf{Z}} \mathcal{L}_{n}^{M}(k+1)
$$


of Aut $F_{n}^{M}$ where $\mathcal{L}_{\mathrm{IA}_{n}^{M}}(k)$ is the $k$-th graded quotient of the lower central series of $\mathrm{IA}_{n}^{M}$, and $\mathcal{L}_{n}^{M}(k)$ is that of $F_{n}^{M}$. In our previous work [22], we studied the Johnson homomorphism of Aut $F_{n}^{M}$ and determined its cokernel. In particular, we showed that there appears only the Morita obstruction $S^{k} H$ in $\operatorname{Coker}\left(\tau_{k}^{\prime}\right)$ for any $k \geq 2$ and $n \geq 4$. We remark that in [22], we determined the cokernel of the Johnson homomorphism $\tau_{k}$ which is defined on the graded quotient of the Johnson filtration of Aut $F_{n}^{M}$. Observing our proof, we verify that $\operatorname{Coker}\left(\tau_{k}^{\prime}\right)=\operatorname{Coker}\left(\tau_{k}\right)$.

Now, similarly to the free group case, we can also construct a $\operatorname{GL}(n, \mathbf{Z})$-equivariant homomorphism

$$
\mu_{k}: Q^{k}\left(\operatorname{IA}_{n}^{M}\right) \rightarrow \operatorname{Hom}_{\mathbf{Z}}\left(H, \alpha_{k+1}\left(\mathcal{L}_{n}^{M}(k+1)\right)\right)
$$

such that $\mu_{k} \circ \alpha_{k}=\alpha_{k+1}^{*} \circ \tau_{k}^{\prime}$. The second purpose of the paper is to show:

Theorem 2 (See Theorem 5.3.) For $k \geq 2$ and $n \geq 4$, the $\operatorname{GL}(n, \mathbf{Z})$-equivariant homomorphism

$$
\mu_{k} \oplus \pi_{k}: Q^{k}\left(\mathrm{IA}_{n}^{M}\right) \rightarrow\left(H^{*} \otimes \mathrm{z} \alpha_{k+1}\left(\mathcal{L}_{n}^{M}(k+1)\right)\right) \oplus S^{k}\left(\left(\mathrm{IA}_{n}^{M}\right)^{\mathrm{ab}}\right)
$$

defined by $\sigma \mapsto\left(\mu_{k}(\sigma), \pi_{k}(\sigma)\right)$ is surjective.

In this paper, for an arbitrary group $G$, we construct a lift of the Johnson homomorphism of the automorphism group of $G$ to the augmentation quotients of $G$. In order to do this, in Section 2, after fixing notation and conventions, we recall the associated graded Lie algebra of a group $G$, the Johnson homomorphism of the automorphism group of $G$, and the associated graded ring of the integral group ring $\mathbf{Z}[G]$ of $G$. In Section 3, we construct an Aut $G / \operatorname{IA}(G)$-equivariant homomorphism $\mu_{k}$ which is considered as a lift of the Johnson homomorphism. In Sections 4 and 5, we consider the case where $G$ is a free group and a free metabelian group respectively.

\section{Preliminaries}

\subsection{Notation and conventions}

Throughout the paper, we use the following notation and conventions. Let $G$ be a group and $N$ a normal subgroup of $G$.

- The abelianization of $G$ is denoted by $G^{\mathrm{ab}}$.

- The group Aut $G$ of $G$ acts on $G$ from the right. For any $\sigma \in$ Aut $G$ and $x \in G$, the action of $\sigma$ on $x$ is denoted by $x^{\sigma}$. 
- For an element $g \in G$, we also denote the coset class of $g$ by $g \in G / N$ if there is no confusion.

- For elements $x$ and $y$ of $G$, the commutator bracket $[x, y]$ of $x$ and $y$ is defined to be $[x, y]:=x y x^{-1} y^{-1}$.

\subsection{Associated graded Lie algebra of a group}

For a group $G$, we define the lower central series of $G$ by the rule

$$
\Gamma_{G}(1):=G, \quad \Gamma_{G}(k):=\left[\Gamma_{G}(k-1), G\right], \quad k \geq 2 .
$$

We denote by $\mathcal{L}_{G}(k):=\Gamma_{G}(k) / \Gamma_{G}(k+1)$ the graded quotient of the lower central series of $G$, and by $\mathcal{L}_{G}:=\bigoplus_{k \geq 1} \mathcal{L}_{G}(k)$ the associated graded sum. The graded sum $\mathcal{L}_{G}$ naturally has a graded Lie algebra structure induced from the commutator bracket on $G$, and called the associated graded Lie algebra of $G$.

For any $g_{1}, \ldots, g_{t} \in G$, a commutator of weight $k$ type of

$$
\left[\left[\cdots\left[\left[g_{i_{1}}, g_{i_{2}}\right], g_{i_{3}}\right], \ldots\right], g_{i_{k}}\right], \quad i_{j} \in\{1, \ldots, t\},
$$

with all of its left brackets to the left of all the elements occurring is called a simple $k$-fold commutator among the components $g_{1}, \ldots, g_{t}$, and we denote it by

$$
\left[g_{i_{1}}, g_{i_{2}}, \ldots, g_{i_{k}}\right]
$$

for simplicity. In general, if $G$ is generated by $g_{1}, \ldots, g_{t}$, then the graded quotient $\mathcal{L}_{G}(k)$ is generated by the simple $k$-fold commutators

$$
\left[g_{i_{1}}, g_{i_{2}}, \ldots, g_{i_{k}}\right], \quad 1 \leq i_{j} \leq t,
$$

as a $\mathbf{Z}$-module.

Let $\rho_{G}$ : Aut $G \rightarrow$ Aut $G^{\text {ab }}$ be the natural homomorphism induced from the abelianization of $G$. The kernel $\operatorname{IA}(G)$ of $\rho_{G}$ is called the IA-automorphism group of $G$. Then the automorphism group Aut $G$ naturally acts on $\mathcal{L}_{G}(k)$ for each $k \geq 1$, and $\operatorname{IA}(G)$ acts on it trivially. Hence the action of $\operatorname{Aut} G / \operatorname{IA}(G)$ on $\mathcal{L}_{G}(k)$ is well-defined.

\subsection{Johnson homomorphisms}

For $k \geq 1$, the action of Aut $G$ on each nilpotent quotient $G / \Gamma_{G}(k+1)$ induces a homomorphism

$$
\text { Aut } G \rightarrow \operatorname{Aut}\left(G / \Gamma_{G}(k+1)\right) \text {. }
$$


For $k=1$, this homomorphism is just $\rho_{G}$. We denote the kernel of the homomorphism above by $\mathcal{A}_{G}(k)$. Then the groups $\mathcal{A}_{G}(k)$ define a descending central filtration

$$
\mathrm{IA}_{G}=\mathcal{A}_{G}(1) \supset \mathcal{A}_{G}(2) \supset \mathcal{A}_{G}(3) \supset \cdots .
$$

(See Andreadakis [1] for details.) We call it the Johnson filtration of Aut $G$. For each $k \geq 1$, the group Aut $G$ acts on $\mathcal{A}_{G}(k)$ by conjugation, and it naturally induces an action of Aut $G / \operatorname{IA}(G)$ on $\operatorname{gr}^{k}\left(\mathcal{A}_{G}\right)$. The graded $\operatorname{sum} \operatorname{gr}\left(\mathcal{A}_{G}\right):=\bigoplus_{k \geq 1} \operatorname{gr}^{k}\left(\mathcal{A}_{G}\right)$ has a graded Lie algebra structure induced from the commutator bracket on $\operatorname{IA}(G)$.

To study the Aut $G / \operatorname{IA}(G)$-module structure of each graded quotient $\operatorname{gr}^{k}\left(\mathcal{A}_{G}\right)$, we define the Johnson homomorphisms of Aut $G$ in the following way. For each $k \geq 1$, we consider a homomorphism $\mathcal{A}_{G}(k) \rightarrow \operatorname{Hom}_{\mathbf{Z}}\left(G^{\mathrm{ab}}, \mathcal{L}_{G}(k+1)\right)$ defined by

$$
\sigma \mapsto\left(g \mapsto g^{-1} g^{\sigma}\right), \quad x \in G .
$$

Then the kernel of this homomorphism is just $\mathcal{A}_{G}(k+1)$. Hence it induces an injective homomorphism

$$
\tau_{k}=\tau_{G, k}: \operatorname{gr}^{k}\left(\mathcal{A}_{G}\right) \hookrightarrow \operatorname{Hom}_{\mathbf{Z}}\left(G^{\mathrm{ab}}, \mathcal{L}_{G}(k+1)\right) .
$$

The homomorphism $\tau_{k}$ is called the $k$-th Johnson homomorphism of Aut $G$. It is easily seen that each $\tau_{k}$ is an Aut $G / \operatorname{IA}(G)$-equivariant homomorphism. Since each Johnson homomorphism $\tau_{k}$ is injective, it is natural question to determine the image, or equivalently, the cokernel of $\tau_{k}$ in the study of the Aut $G / \operatorname{IA}(G)$-module $\operatorname{gr}^{k}\left(\mathcal{A}_{G}\right)$.

Here, we consider another descending filtration of $\operatorname{IA}(G)$. Let $\Gamma_{\operatorname{IA}(G)}(k)$ be the $k$-th subgroup of the lower central series of $\operatorname{IA}(G)$. Then for each $k \geq 1, \Gamma_{\operatorname{IA}(G)}(k)$ is a subgroup of $\mathcal{A}_{G}(k)$ since the Johnson filtration is a central filtration of $\operatorname{IA}(G)$. In general, it is a natural question to ask whether $\Gamma_{\mathrm{IA}(G)}(k)$ coincides with $\mathcal{A}_{G}(k)$ or not. For the case where $G$ is a free group $F_{n}$ of rank $n$, it is conjectured that $\Gamma_{\operatorname{IA}\left(F_{n}\right)}(k)$ coincides with $\mathcal{A}_{F_{n}}(k)$ by Andreadakis.

Consider $\mathcal{L}_{\mathrm{IA}(G)}(k):=\Gamma_{\mathrm{IA}(G)}(k) / \Gamma_{\mathrm{IA}(G)}(k+1)$ for each $k \geq 1$. Similarly to $\operatorname{gr}\left(\mathcal{A}_{G}\right)$, the graded sum $\mathcal{L}_{\mathrm{IA}(G)}:=\bigoplus_{k \geq 1} \mathcal{L}_{\mathrm{IA}(G)}(k)$ has a graded Lie algebra structure induced from the commutator bracket on $\operatorname{IA}(G)$. The restriction of the homomorphism $\mathcal{A}_{G}(k) \rightarrow \operatorname{Hom}_{\mathbf{Z}}\left(G^{\mathrm{ab}}, \mathcal{L}_{G}(k+1)\right)$ to $\Gamma_{\mathrm{IA}(G)}(k)$ also induces an $\operatorname{Aut} G / \operatorname{IA}(G)-$ equivariant homomorphism

$$
\tau_{k}^{\prime}=\tau_{G, k}^{\prime}: \mathcal{L}_{\mathrm{IA}(G)}(k) \rightarrow \operatorname{Hom}_{\mathbf{Z}}\left(G^{\mathrm{ab}}, \mathcal{L}_{G}(k+1)\right) .
$$

In this paper, we also call $\tau_{k}^{\prime}$ the $k$-th Johnson homomorphism of Aut $G$. 


\subsection{Associated graded ring of a group ring}

For a group $G$, let $\mathbf{Z}[G]$ be a group ring of $G$ over $\mathbf{Z}$, and $\Delta(G)$ the augmentation ideal of $\mathbf{Z}[G]$. Namely, $\Delta(G)$ is the kernel of the augmentation map $\varepsilon: \mathbf{Z}[G] \rightarrow \mathbf{Z}$ defined by

$$
\sum_{g \in G} a_{g} g \mapsto \sum_{g \in G} a_{g}, \quad a_{g} \in \mathbf{Z} .
$$

We denote by $\Delta^{k}(G):=(\Delta(G))^{k}$ the $k$-times product of the augmentation ideal $\Delta(G)$ in $\mathbf{Z}[G]$. For each $k \geq 1$, set

$$
\begin{aligned}
Q^{k}(G) & :=\Delta^{k}(G) / \Delta^{k+1}(G), \\
\operatorname{gr}(\mathbf{Z}[G]) & :=\bigoplus_{k \geq 1} Q^{k}(G) .
\end{aligned}
$$

The quotients $Q^{k}(G)$ are called the augmentation quotients of $G$. The graded sum $\operatorname{gr}(\mathbf{Z}[G])$ naturally has an associative graded ring structure induced from the product in $\mathbf{Z}[G]$. The ring $\operatorname{gr}(\mathbf{Z}[G])$ is called the associated graded ring of the group ring $\mathbf{Z}[G]$.

In general, one of the most standard methods to study $Q^{k}(G)$ is to consider a natural surjective homomorphism $\pi_{k}=\pi_{k, G}: Q^{k}(G) \rightarrow Q^{k}\left(G^{\mathrm{ab}}\right)$ induced from the abelianization $G \rightarrow G^{\mathrm{ab}}$. Furthermore, if $G^{\mathrm{ab}}$ is free abelian, we have an natural isomorphism $Q^{k}\left(G^{\mathrm{ab}}\right) \cong S^{k}\left(G^{\mathrm{ab}}\right)=S^{k}\left(\mathcal{L}_{G}(1)\right)$ where $S^{k}$ means the $k$-th symmetric power. (See Passi [17, Corollary 8.2].) In Section 4.2, we study the kernel of $\pi_{k}$ for $G=F_{n}$. We remark that for a group $G$ and $k \geq 1, \operatorname{Ker}\left(\pi_{k}\right)$ is generated by elements

$$
\left(g_{1}-1\right) \cdots\left(g_{k}-1\right)-\left(g_{\sigma(1)}-1\right) \cdots\left(g_{\sigma(k)}-1\right)
$$

as a $\mathbf{Z}$-module for any $g_{1}, \ldots, g_{k} \in G$ and $\sigma \in \mathfrak{S}_{k}$. Here $\mathfrak{S}_{k}$ denotes the symmetric group of degree $k$.

Here we consider a relation between $\operatorname{gr}(\mathbf{Z}[G])$ and $\mathcal{L}_{G}$. For any $g \in \Gamma_{G}(k)$, it is well known that an element $g-1 \in \mathbf{Z}[G]$ belongs to $\Delta^{k}(G)$. Then a map $\Gamma_{G}(k) \rightarrow \Delta^{k}(G)$ defined by $g \mapsto g-1$ induces a $\mathbf{Z}$-linear map

$$
\alpha_{k}=\alpha_{k, G}: \mathcal{L}_{G}(k) \rightarrow Q^{k}(G)
$$

and a Lie algebra homomorphism

$$
\alpha_{G}:=\bigoplus_{k \geq 1} \alpha_{k}: \mathcal{L}_{G} \rightarrow \operatorname{gr}(\mathbf{Z}[G])
$$

where we consider $\operatorname{gr}(\mathbf{Z}[G])$ as a Lie algebra with a Lie bracket $[x, y]:=x y-y x$ for any $x, y \in \mathbf{Z}[G]$. We remark that for any group $G, \alpha_{1, G}: G^{\text {ab }} \rightarrow Q^{1}(G)$ is an 
isomorphism. Hence, so is $\pi_{1}$. For $k \geq 2$, however, $\pi_{k}$ is not injective in general. For $k=2$, if $G$ is a finitely generated, then we have a split exact sequence of $\mathbf{Z}$-modules:

$$
0 \rightarrow \mathcal{L}_{G}(2) \stackrel{\alpha_{2, G}}{\longrightarrow} Q^{2}(G) \stackrel{\pi_{2, G}}{\longrightarrow} Q^{2}\left(G^{\mathrm{ab}}\right) \rightarrow 0 .
$$

(For a proof, see [17, Corollary 8.13, Chapter VIII].) We denote by

$$
\alpha_{k+1}^{*}=\alpha_{k+1, G}^{*}: \operatorname{Hom}_{\mathbf{Z}}\left(G^{\mathrm{ab}}, \mathcal{L}_{G}(k+1)\right) \rightarrow \operatorname{Hom}_{\mathbf{Z}}\left(G^{\mathrm{ab}}, Q^{k+1}(G)\right)
$$

the natural homomorphism induced from $\alpha_{k+1}$.

\section{A lift of the Johnson homomorphisms to the augmentation quotients}

In this section, for a group $G$, we construct an Aut $G / \operatorname{IA}(G)$-equivariant homomorphism $\mu_{k}: Q^{k}(\operatorname{IA}(G)) \rightarrow \operatorname{Hom}_{\mathbf{Z}}\left(G^{\mathrm{ab}}, Q^{k+1}(G)\right)$ such that

$$
\mu_{k} \circ \alpha_{k, \mathrm{IA}(G)}=\alpha_{k+1, G}^{*} \circ \tau_{k}^{\prime} \text {. }
$$

\subsection{Construction of $\mu_{k}$}

For any $\sigma \in$ Aut $G$ and $x \in G$, set $s_{\sigma}(x):=x^{-1} x^{\sigma} \in G$. First, we recall an important and useful lemma due to Andreadakis [1]:

Lemma 3.1 For any $k, l \geq 1, \sigma \in \mathcal{A}_{G}(k)$ and $x \in \Gamma_{G}(l)$, we have $s_{\sigma}(x) \in \Gamma_{G}(k+l)$.

For the proof of Lemma 3.1, see [1]. From this lemma, we see that $s_{\sigma}(x)-1 \in \Delta^{k+l}(G)$ for any $\sigma \in \mathcal{A}_{G}(k)$ and $x \in \Gamma_{G}(l)$. We often use these facts without any quotation. In order to define a lift of the Johnson homomorphism, we prepare some lemmas.

Lemma 3.2 For any $\sigma, \tau \in \operatorname{IA}(G)$ and $x, y \in G$, we have

(1) $s_{\sigma \tau}(x)=s_{\tau}(x) \cdot s_{\sigma}(x)^{\tau}=s_{\tau}(x) s_{\sigma}(x) s_{\tau}\left(s_{\sigma}(x)\right)$.

(2) $s_{\sigma}(x y)=y^{-1} s_{\sigma}(x) y \cdot s_{\sigma}(y)=\left[y^{-1}, s_{\sigma}(x)\right] s_{\sigma}(x) s_{\sigma}(y)$.

Proof The equations follow from

$$
\begin{aligned}
& s_{\sigma \tau}(x)=x^{-1} x^{\sigma \tau}=x^{-1} x^{\tau} \cdot\left(x^{-1} x^{\sigma}\right)^{\tau}=x^{-1} x^{\tau} \cdot x^{-1} x^{\sigma} \cdot\left(x^{-1} x^{\sigma}\right)^{-1} \cdot\left(x^{-1} x^{\sigma}\right)^{\tau}, \\
& s_{\sigma}(x y)=y^{-1} x^{-1} x^{\sigma} y^{\sigma}=y^{-1} x^{-1} x^{\sigma} y \cdot y^{-1} y^{\sigma} .
\end{aligned}
$$

Lemma 3.3 For any $x \in \Gamma_{G}(k)$ and $\sigma \in \operatorname{IA}(G)$, we have

$$
x^{\sigma}-x \equiv s_{\sigma}(x)-1 \quad\left(\bmod \Delta^{k+2}(G)\right) .
$$


Proof This is clear from

$$
\begin{aligned}
x^{\sigma}-x & =\left(x^{\sigma}-1\right)-(x-1) \\
& =\left(x\left(x^{-1} x^{\sigma}\right)-1\right)-(x-1) \\
& =(x-1)\left(s_{\sigma}(x)-1\right)+\left(s_{\sigma}(x)-1\right)
\end{aligned}
$$

as $s_{\sigma}(x)-1 \in \Delta^{k+1}(G)$, and hence $(x-1)\left(s_{\sigma}(x)-1\right) \in \Delta^{k+2}(G)$.

Lemma 3.4 For any $a \in \Delta^{k}(G)$ and $\sigma \in \operatorname{IA}(G)$, we have $a^{\sigma}-a \in \Delta^{k+1}(G)$.

Proof Any element of $\Delta^{k}(G)$ can be written as a $\mathbf{Z}$-linear combination of elements types of

$$
\left(x_{1}-1\right) \cdots\left(x_{k}-1\right)
$$

for $x_{i} \in G$. Hence it suffices to show the lemma for $a=\left(x_{1}-1\right) \cdots\left(x_{k}-1\right)$. Then we have

$$
\begin{aligned}
& a^{\sigma}-a=\left(x_{1}\left(x_{1}^{-1} x_{1}^{\sigma}\right)-1\right) \cdots\left(x_{k}\left(x_{k}^{-1} x_{k}^{\sigma}\right)-1\right)-\left(x_{1}-1\right) \cdots\left(x_{k}-1\right) \\
&=\left\{\left(x_{1}-1\right)\left(x_{1}^{-1} x_{1}^{\sigma}-1\right)+\left(x_{1}-1\right)+\left(x_{1}^{-1} x_{1}^{\sigma}-1\right)\right\} \\
& \cdots\left\{\left(x_{k}-1\right)\left(x_{k}^{-1} x_{k}^{\sigma}-1\right)+\left(x_{k}-1\right)+\left(x_{k}^{-1} x_{k}^{\sigma}-1\right)\right\}-\left(x_{1}-1\right) \cdots\left(x_{k}-1\right) \\
& \equiv\left(x_{1}-1\right) \cdots\left(x_{k}-1\right)-\left(x_{1}-1\right) \cdots\left(x_{k}-1\right)=0 \quad\left(\bmod \Delta^{k+1}(G)\right) .
\end{aligned}
$$

For any $x \in G$, consider a $\mathbf{Z}$-linear homomorphism $\varphi_{x}: \mathbf{Z}[\operatorname{IA}(G)] \rightarrow \Delta(G)$ defined by $\sigma \mapsto s_{\sigma}(x)-1$ for any $\sigma \in \operatorname{IA}(G)$.

Lemma 3.5 For any $k, l \geq 1, x \in \Gamma_{G}(l)$, and $\sigma_{1}, \ldots, \sigma_{k} \in \operatorname{IA}(G)$, we have

$$
\varphi_{x}\left(\left(\sigma_{1}-1\right) \cdots\left(\sigma_{k}-1\right)\right) \equiv s_{\sigma_{k}}\left(s_{\sigma_{k-1}}\left(\cdots\left(s_{\sigma_{1}}(x)\right) \cdots\right)\right)-1 \quad\left(\bmod \Delta^{k+l+1}(G)\right) .
$$

Proof We prove this lemma by the induction on $k \geq 1$. For $k=1$, it is obvious by the definition. Assume that $k \geq 2$. Write

$$
\left(\sigma_{1}-1\right) \cdots\left(\sigma_{k-1}-1\right)=\sum_{\sigma \in \operatorname{IA}(G)} a_{\sigma} \sigma \in \mathbf{Z}[\operatorname{IA}(G)]
$$


for $a_{\sigma} \in \mathbf{Z}$. Then we have

$$
\begin{aligned}
\varphi_{x}\left(\left(\sigma_{1}-1\right) \cdots\left(\sigma_{k-1}-1\right)\left(\sigma_{k}-1\right)\right), \\
\quad=\varphi_{x}\left(\left(\sigma_{1}-1\right) \cdots\left(\sigma_{k-1}-1\right) \sigma_{k}-\left(\sigma_{1}-1\right) \cdots\left(\sigma_{k-1}-1\right)\right), \\
=\varphi_{x}\left(\sum_{\sigma \in \operatorname{IA}(G)} a_{\sigma} \sigma \sigma_{k}-\sum_{\sigma \in \operatorname{IA}(G)} a_{\sigma} \sigma\right), \\
=\sum_{\sigma \in \operatorname{IA}(G)} a_{\sigma}\left\{\left(s_{\sigma \sigma_{k}}(x)-1\right)-\left(s_{\sigma}(x)-1\right)\right\}, \\
=\sum_{\sigma \in \operatorname{IA}(G)} a_{\sigma}\left\{\left(s_{\sigma_{k}}(x) s_{\sigma}(x)^{\sigma_{k}}-1\right)-\left(s_{\sigma}(x)-1\right)\right\}, \\
=\sum_{\sigma \in \operatorname{IA}(G)} a_{\sigma}\left\{\left(s_{\sigma_{k}}(x)-1\right)\left(s_{\sigma}(x)^{\sigma_{k}}-1\right)+\left(s_{\sigma_{k}}(x)-1\right)\right. \\
\left.\quad+\left(s_{\sigma}(x)^{\sigma_{k}}-1\right)-\left(s_{\sigma}(x)-1\right)\right\} .
\end{aligned}
$$

Here we see

$$
\begin{aligned}
\sum_{\sigma \in \mathrm{IA}(G)} a_{\sigma}\left(s_{\sigma_{k}}(x)-1\right)\left(s_{\sigma}(x)^{\sigma_{k}}-1\right) & =\left(s_{\sigma_{k}}(x)-1\right)\left(\sum_{\sigma \in \mathrm{IA}(G)} a_{\sigma}\left(s_{\sigma}(x)-1\right)\right)^{\sigma_{k}} \\
& \equiv 0 \quad\left(\bmod \Delta^{k+l+1}(G)\right)
\end{aligned}
$$

since $s_{\sigma_{k}}(x)-1 \in \Delta^{2}(G)$ and $\sum_{\sigma \in \mathrm{IA}(G)} a_{\sigma}\left(s_{\sigma}(x)-1\right) \in \Delta^{k+l-1}(G)$ by the inductive hypothesis, and see

$$
\sum_{\sigma \in \operatorname{IA}(G)} a_{\sigma}\left(s_{\sigma_{k}}(x)-1\right)=\left(s_{\sigma_{k}}(x)-1\right) \sum_{\sigma \in \operatorname{IA}(G)} a_{\sigma}=0 .
$$

On the other hand, by the inductive hypothesis, we have

$$
\begin{aligned}
\sum_{\sigma \in \operatorname{IA}(G)} a_{\sigma}\left\{\left(s_{\sigma}(x)^{\sigma_{k}}-1\right)-\left(s_{\sigma}(x)-1\right)\right\} \\
\quad=\left(\sum_{\sigma \in \operatorname{IA}(G)} a_{\sigma}\left(s_{\sigma}(x)-1\right)\right)^{\sigma_{k}}-\sum_{\sigma \in \operatorname{IA}(G)} a_{\sigma}\left(s_{\sigma}(x)-1\right) \\
\quad=\left(s_{\sigma_{k-1}}\left(\cdots\left(s_{\sigma_{1}}(x)\right) \cdots\right)-1\right)^{\sigma_{k}}-\left(s_{\sigma_{k-1}}\left(\cdots\left(s_{\sigma_{1}}(x)\right) \cdots\right)-1\right)+a^{\sigma_{k}}-a
\end{aligned}
$$

for some $a \in \Delta^{k+l}(G)$. Then, by Lemmas 3.3 and 3.4, we see this is congruent to

$$
s_{\sigma_{k}}\left(s_{\sigma_{k-1}}\left(\cdots\left(s_{\sigma_{1}}(x)\right) \cdots\right)\right)-1 \quad\left(\bmod \Delta^{k+l+1}(G)\right) .
$$

This completes the proof of Lemma 3.5. 
For each $k \geq 1$, since $\Delta^{k}(\operatorname{IA}(G))$ is generated by elements types of

$$
\left(\sigma_{1}-1\right) \cdots\left(\sigma_{k}-1\right)
$$

for $\sigma_{i} \in \operatorname{IA}(G)$ as a $\mathbf{Z}$-module, by Lemma 3.5 we obtain:

Corollary 3.6 For any $k, l \geq 1$ and $x \in \Gamma_{G}(l)$, we have

$$
\varphi_{x}\left(\Delta^{k}(\operatorname{IA}(G))\right) \subset \Delta^{k+l}(\operatorname{IA}(G)) .
$$

Remark 3.7 For any $x \in \Gamma_{G}(l)$ a homomorphism $\mathbf{Z}[\operatorname{IA}(G)] \rightarrow Q^{k+l}(\operatorname{IA}(G))$ defined by $a \mapsto \varphi_{x}(a)$ is a polynomial map of degree $\leq k$. (For details for polynomial maps, see Passi [17], for example.)

Lemma 3.8 For any $k, l \geq 1$ and $x, y \in \Gamma_{G}(l)$, we have $s_{\sigma_{k}}\left(\cdots\left(s_{\sigma_{1}}(x y)\right) \cdots\right) \equiv s_{\sigma_{k}}\left(\cdots\left(s_{\sigma_{1}}(x)\right) \cdots\right) \cdot s_{\sigma_{k}}\left(\cdots\left(s_{\sigma_{1}}(y)\right) \cdots\right)\left(\bmod \Gamma_{G}(k+2 l)\right)$ for any $\sigma_{1}, \ldots, \sigma_{k} \in \operatorname{IA}(G)$.

Proof We prove this lemma by the induction on $k \geq 1$. If $k=1$, it is trivial from the part (2) of Lemma 3.2. Assume $k \geq 2$. By the inductive hypothesis, we see

$$
s_{\sigma_{k-1}}\left(\cdots\left(s_{\sigma_{1}}(x y)\right)\right)=c s_{\sigma_{k-1}}\left(\cdots\left(s_{\sigma_{1}}(x)\right)\right) \cdot s_{\sigma_{k-1}}\left(\cdots\left(s_{\sigma_{1}}(y)\right)\right)
$$

for some $c \in \Gamma_{G}(k+2 l-1)$. Then, using the part (2) of Lemma 3.2 we have

$$
\begin{aligned}
& s_{\sigma_{k}}\left(s_{\sigma_{k-1}}\left(\cdots\left(s_{\sigma_{1}}(x y)\right)\right)\right) \\
& \quad=s_{\sigma_{k}}\left(c s_{\sigma_{k-1}}\left(\cdots\left(s_{\sigma_{1}}(x)\right)\right) \cdot s_{\sigma_{k-1}}\left(\cdots\left(s_{\sigma_{1}}(y)\right)\right)\right) \\
& =\left[\left\{s_{\sigma_{k-1}}\left(\cdots\left(s_{\sigma_{1}}(x)\right)\right) s_{\sigma_{k-1}}\left(\cdots\left(s_{\sigma_{1}}(y)\right)\right)\right\}^{-1}, s_{\sigma_{k}}(c)\right] \\
& \quad \cdot s_{\sigma_{k}}(c) \cdot s_{\sigma_{k}}\left(s_{\sigma_{k-1}}\left(\cdots\left(s_{\sigma_{1}}(x)\right)\right) \cdot s_{\sigma_{k-1}}\left(\cdots\left(s_{\sigma_{1}}(y)\right)\right)\right) \\
& \equiv s_{\sigma_{k}}\left(s_{\sigma_{k-1}}\left(\cdots\left(s_{\sigma_{1}}(x)\right)\right) \cdot s_{\sigma_{k-1}}\left(\cdots\left(s_{\sigma_{1}}(y)\right)\right)\right), \\
& \left.=\left[s_{\sigma_{k-1}}\left(\cdots\left(s_{\sigma_{1}}(y)\right)\right)\right)^{-1}, s_{\sigma_{k}}\left(s_{\sigma_{k-1}}\left(\cdots\left(s_{\sigma_{1}}(x)\right)\right)\right)\right] \\
& \quad \cdot s_{\sigma_{k}}\left(s_{\sigma_{k-1}}\left(\cdots\left(s_{\sigma_{1}}(x)\right)\right)\right) \cdot s_{\sigma_{k}}\left(s_{\sigma_{k-1}}\left(\cdots\left(s_{\sigma_{1}}(y)\right)\right)\right) \\
& \equiv s_{\sigma_{k}}\left(s_{\sigma_{k-1}}\left(\cdots\left(s_{\sigma_{1}}(x)\right)\right)\right) \cdot s_{\sigma_{k}}\left(s_{\sigma_{k-1}}\left(\cdots\left(s_{\sigma_{1}}(y)\right)\right)\right) .
\end{aligned}
$$

modulo $\Gamma_{G}(k+2 l)$.

Lemma 3.9 For any $k, l \geq 1, x, y \in \Gamma_{G}(l)$, and $a \in \Delta^{k}(\operatorname{IA}(G))$, we have

$$
\varphi_{x y}(a) \equiv \varphi_{x}(a)+\varphi_{y}(a) \quad\left(\bmod \Delta^{k+l+1}(G)\right) .
$$


Proof First, we consider the case where $a=\left(\sigma_{1}-1\right) \cdots\left(\sigma_{k}-1\right)$ for some $\sigma_{i} \in \operatorname{IA}(G)$. From Lemma 3.5 and Lemma 3.8, we see

$$
\begin{aligned}
\varphi_{x y}(a) & \equiv s_{\sigma_{k}}\left(s_{\sigma_{k-1}}\left(\cdots\left(s_{\sigma_{1}}(x y)\right) \cdots\right)\right)-1 \\
& =c s_{\sigma_{k}}\left(\cdots\left(s_{\sigma_{1}}(x)\right) \cdots\right) \cdot s_{\sigma_{k}}\left(\cdots\left(s_{\sigma_{1}}(y)\right) \cdots\right)-1
\end{aligned}
$$

for some $c \in \Gamma_{G}(k+2 l)$. Hence we have

$$
\begin{aligned}
\varphi_{x y}(a)= & (c-1)\left(s_{\sigma_{k}}\left(\cdots\left(s_{\sigma_{1}}(x)\right) \cdots\right) \cdot s_{\sigma_{k}}\left(\cdots\left(s_{\sigma_{1}}(y)\right) \cdots\right)-1\right) \\
& \quad+(c-1)+\left(s_{\sigma_{k}}\left(\cdots\left(s_{\sigma_{1}}(x)\right) \cdots\right) \cdot s_{\sigma_{k}}\left(\cdots\left(s_{\sigma_{1}}(y)\right) \cdots\right)-1\right) \\
\equiv & s_{\sigma_{k}}\left(\cdots\left(s_{\sigma_{1}}(x)\right) \cdots\right) \cdot s_{\sigma_{k}}\left(\cdots\left(s_{\sigma_{1}}(y)\right) \cdots\right)-1 \\
= & \left(s_{\sigma_{k}}\left(\cdots\left(s_{\sigma_{1}}(x)\right) \cdots\right)-1\right)\left(s_{\sigma_{k}}\left(\cdots\left(s_{\sigma_{1}}(y)\right) \cdots\right)-1\right) \\
& \quad+\left(s_{\sigma_{k}}\left(\cdots\left(s_{\sigma_{1}}(x)\right) \cdots\right)-1\right)+\left(s_{\sigma_{k}}\left(\cdots\left(s_{\sigma_{1}}(y)\right) \cdots\right)-1\right) \\
\equiv & \left(s_{\sigma_{k}}\left(\cdots\left(s_{\sigma_{1}}(x)\right) \cdots\right)-1\right)+\left(s_{\sigma_{k}}\left(\cdots\left(s_{\sigma_{1}}(y)\right) \cdots\right)-1\right) \\
= & \varphi_{x}(a)+\varphi_{y}(a)
\end{aligned}
$$

modulo $\Delta^{k+l+1}(G)$.

For a general case, $a \in \Delta^{k}(\operatorname{IA}(G))$ is written as a $\mathbf{Z}$-linear combination of elements types of

$$
\left(\sigma_{1}-1\right) \cdots\left(\sigma_{k}-1\right)
$$

Therefore, using the argument above, we obtain the lemma for any $a \in \Delta^{k}(\operatorname{IA}(G))$.

Lemma 3.10 For any $a \in \Delta^{k}(\operatorname{IA}(G))$, a map $\mu_{k}(a): G^{\mathrm{ab}} \rightarrow Q^{k+1}(G)$ defined by $x \mapsto \varphi_{x}(a)$ is a homomorphism.

Proof To begin with, we check that $\mu_{k}(a)$ is well-defined. Consider elements $x, y \in G$ such that $y=x c$ for some $c \in \Gamma_{G}(2)$. Then by Lemma 3.9,

$$
\varphi_{y}(a)=\varphi_{x c}(a) \equiv \varphi_{x}(a)+\varphi_{c}(a) \quad\left(\bmod \Delta^{k+2}(G)\right) .
$$

On the other hand, by Corollary 3.6, we see $\varphi_{c}(a) \in \Delta^{k+2}(G)$. Hence $\varphi_{y}(a)=$ $\varphi_{x}(a) \in Q^{k+1}(G)$.

To show $\mu_{k}(a)$ is a homomorphism, take any $x$ and $y \in G$. Then by Lemma 3.9,

$$
\mu_{k}(a)(x y)=\varphi_{x y}(a) \equiv \varphi_{x}(a)+\varphi_{y}(a)=\mu_{k}(a)(x)+\mu_{k}(a)(y)
$$

modulo $\Delta^{k+2}(G)$. 
Now, we are ready to define a lift of the Johnson homomorphism $\tau_{k}^{\prime}$. For any $k \geq 1$, define a map

by

$$
\mu_{k}: \Delta^{k}(\operatorname{IA}(G)) \rightarrow \operatorname{Hom}_{\mathbf{Z}}\left(G^{\mathrm{ab}}, Q^{k+1}(G)\right)
$$

$$
a \mapsto\left(x \mapsto \varphi_{x}(a)\right) .
$$

Using Lemma 3.3, it is easy to check that the map $\mu_{k}$ is a homomorphism. Furthermore $\Delta^{k+1}(\operatorname{IA}(G))$ is contained in $\operatorname{Ker}\left(\mu_{k}\right)$. Hence $\mu_{k}$ induces a homomorphism

$$
Q^{k}(\operatorname{IA}(G)) \rightarrow \operatorname{Hom}_{\mathbf{Z}}\left(G^{\mathrm{ab}}, Q^{k+1}(G)\right) .
$$

We also denote by $\mu_{k}$ this induced homomorphism, and call it the $k$-th Johnson homomorphism of $\mathbf{Z}[\operatorname{IA}(G)]$. We see that the compatibility (2) follows by the definition of $\tau_{k}^{\prime}$ and $\mu_{k}$.

\subsection{Actions of Aut $G$}

Next we consider actions of Aut $G$. Since $\operatorname{IA}(G)$ is a normal subgroup of Aut $G$, the group Aut $G$ acts on $\mathbf{Z}[\operatorname{IA}(G)]$ from the right by

$$
\left(\sum_{\sigma \in \mathrm{IA}(G)} a_{\sigma} \sigma\right) \cdot \tau:=\sum_{\sigma \in \mathrm{IA}(G)} a_{\sigma}\left(\tau^{-1} \sigma \tau\right)
$$

for any $\tau \in$ Aut $G$. For each $k \geq 1$, since $\Delta^{k}(\operatorname{IA}(G))$ is preserved by the action of Aut $G$, the group Aut $G$ also acts on each of the graded quotient $Q^{k}(\operatorname{IA}(G))$. Then $\operatorname{IA}(G)$ acts on $Q^{k}(\operatorname{IA}(G))$ trivially. In fact, for any $\tau \in \operatorname{IA}(G)$, we have

$$
\begin{aligned}
\left(\sigma_{1}-1\right) \cdots\left(\sigma_{k}-1\right) \cdot \tau= & \left(\tau^{-1} \sigma_{1} \tau-1\right) \cdots\left(\tau^{-1} \sigma_{k} \tau-1\right) \\
= & \left(\left[\tau^{-1}, \sigma_{1}\right] \sigma_{1}-1\right) \cdots\left(\left[\tau^{-1}, \sigma_{k}\right] \sigma_{k} \tau-1\right) \\
= & \left\{\left(\left[\tau^{-1}, \sigma_{1}\right]-1\right)\left(\sigma_{1}-1\right)+\left(\left[\tau^{-1}, \sigma_{1}\right]-1\right)+\left(\sigma_{1}-1\right)\right\} \\
& \cdots\left\{\left(\left[\tau^{-1}, \sigma_{k}\right]-1\right)\left(\sigma_{k}-1\right)+\left(\left[\tau^{-1}, \sigma_{k}\right]-1\right)+\left(\sigma_{k}-1\right)\right\} \\
\equiv & \left(\sigma_{1}-1\right) \cdots\left(\sigma_{k}-1\right)
\end{aligned}
$$

modulo $\Delta^{k+1}(\operatorname{IA}(G))$ since $\left[\tau^{-1}, \sigma_{i}\right] \in \Gamma_{\operatorname{IA}(G)}(2)$ and $\left[\tau^{-1}, \sigma_{i}\right]-1 \in \Delta^{2}(\operatorname{IA}(G))$. Since $Q^{k}(\operatorname{IA}(G))$ is generated by elements $\left(\sigma_{1}-1\right) \cdots\left(\sigma_{k}-1\right)$ for $\sigma_{i} \in \operatorname{IA}(G)$ as a $\mathbf{Z}$-module, we verify that the action of $\operatorname{IA}(G)$ on $Q^{k}(\operatorname{IA}(G))$ is trivial. Hence the quotient group Aut $G / \operatorname{IA}(G)$ naturally acts on each of $Q^{k}(\operatorname{IA}(G))$ from the right.

Now, Aut $G$ naturally acts on $\operatorname{Hom}_{\mathbf{Z}}\left(G^{\mathrm{ab}}, Q^{k+1}(G)\right)$. Then it is easily seen that the action of $\operatorname{IA}(G)$ on $\operatorname{Hom}_{\mathbf{Z}}\left(G^{\mathrm{ab}}, Q^{k+1}(G)\right)$ is trivial. Hence the quotient group Aut $G / \operatorname{IA}(G)$ also acts on it. To show that $\mu_{k}$ is Aut $G / \operatorname{IA}(G)$-equivariant, we prepare: 
Lemma 3.11 For any $k \geq 1$, and $\sigma, \sigma_{1}, \ldots, \sigma_{k} \in$ Aut $G$, we have

$$
\left(s_{\sigma_{k}}\left(\cdots\left(s_{\sigma_{1}}(x)\right) \cdots\right)\right)^{\sigma}=s_{\sigma^{-1} \sigma_{k} \sigma}\left(\cdots\left(s_{\sigma^{-1} \sigma_{1} \sigma}\left(x^{\sigma}\right)\right) \cdots\right) .
$$

Proof We prove this lemma by the induction on $k \geq 1$. For $k=1$, it is clear by

$$
s_{\sigma_{1}}(x)^{\sigma}=\left(x^{-1} x^{\sigma_{1}}\right)^{\sigma}=\left(x^{\sigma}\right)^{-1} x^{\sigma_{1} \sigma}=\left(x^{\sigma}\right)^{-1}\left(x^{\sigma}\right)^{\sigma^{-1} \sigma_{1} \sigma}=s_{\sigma^{-1} \sigma_{1} \sigma}\left(x^{\sigma}\right) .
$$

Assume $k \geq 2$. Using the inductive hypothesis, we obtain

$$
\begin{aligned}
& \left(s_{\sigma_{k}}\left(\cdots\left(s_{\sigma_{1}}(x)\right) \cdots\right)\right)^{\sigma} \\
& =\left(\left(s_{\sigma_{k-1}}\left(\cdots\left(s_{\sigma_{1}}(x)\right) \cdots\right)\right)^{-1}\left(s_{\sigma_{k-1}}\left(\cdots\left(s_{\sigma_{1}}(x)\right) \cdots\right)\right)^{\sigma_{k}}\right)^{\sigma} \\
& =\left\{\left(s_{\sigma_{k-1}}\left(\cdots\left(s_{\sigma_{1}}(x)\right) \cdots\right)\right)^{\sigma}\right\}^{-1}\left\{\left(s_{\sigma_{k-1}}\left(\cdots\left(s_{\sigma_{1}}(x)\right) \cdots\right)\right)^{\sigma}\right\}^{\sigma^{-1} \sigma_{k} \sigma} \\
& =\left\{s_{\sigma^{-1} \sigma_{k-1} \sigma}\left(\cdots\left(s_{\sigma^{-1} \sigma_{1} \sigma}\left(x^{\sigma}\right)\right) \cdots\right)\right\}^{-1}\left\{s_{\sigma^{-1} \sigma_{k-1} \sigma}\left(\cdots\left(s_{\sigma^{-1} \sigma_{1} \sigma}\left(x^{\sigma}\right)\right) \cdots\right)\right\}^{\sigma^{-1} \sigma_{k} \sigma} \\
& =s_{\sigma^{-1} \sigma_{k} \sigma}\left(\cdots\left(s_{\sigma^{-1}} \sigma_{1} \sigma\left(x^{\sigma}\right)\right) \cdots\right) .
\end{aligned}
$$

This completes the proof of Lemma 3.11.

Proposition 3.12 For any $k \geq 1$, the Johnson homomorphism $\mu_{k}$ is an Aut $G / \operatorname{IA}(G)$ equivariant homomorphism.

Proof It is enough to show that $\mu_{k}\left(a^{\sigma}\right)=\left(\mu_{k}(a)\right)^{\sigma}$ for $\sigma \in \operatorname{IA}(G)$ and $a=$ $\left(\sigma_{1}-1\right) \cdots\left(\sigma_{k}-1\right) \in Q^{k}(\operatorname{IA}(G))$. Then, for any $x \in G^{\mathrm{ab}}$ we have

$$
\begin{aligned}
\mu_{k}\left(a^{\sigma}\right)(x) & =\mu_{k}\left(\left(\sigma^{-1} \sigma_{1} \sigma-1\right) \cdots\left(\sigma^{-1} \sigma_{k} \sigma-1\right)\right)(x) \\
& =s_{\sigma^{-1}} \sigma_{k} \sigma\left(\cdots\left(s_{\sigma^{-1}} \sigma_{1} \sigma(x)\right) \cdots\right)-1 .
\end{aligned}
$$

On the other hand, by Lemma 3.11 ,

$$
\begin{aligned}
\left(\mu_{k}(a)\right)^{\sigma}(x) & =\left(\mu_{k}(a)\left(x^{\sigma^{-1}}\right)\right)^{\sigma}=\left(s_{\sigma_{k}}\left(\cdots\left(s_{\sigma_{1}}\left(x^{\sigma^{-1}}\right)\right) \cdots\right)-1\right)^{\sigma} \\
& =s_{\sigma^{-1} \sigma_{k} \sigma}\left(\cdots\left(s_{\sigma^{-1} \sigma_{1} \sigma}(x)\right) \cdots\right)-1 .
\end{aligned}
$$

for any $x \in G^{\mathrm{ab}}$.

\subsection{Some properties of $\mu_{k}$}

Here we observe some properties of $\mu_{k}$. First, we consider the image of $\mu_{k}$. In general, $\mu_{k}$ is not surjective.

Lemma 3.13 For each $k \geq 1$, the image of $\mu_{k}$ is contained in that of $\alpha_{k+1, G}^{*}$. 
Proof Since $Q^{k}(\operatorname{IA}(G))$ is generated by $\left(\sigma_{1}-1\right) \cdots\left(\sigma_{k}-1\right)$ for $\sigma_{i} \in \operatorname{IA}(G)$ as a $\mathbf{Z}$-module, it suffices to show $\mu_{k}(a) \in \operatorname{Im}\left(\alpha_{k+1, G}^{*}\right)$ for $a=\left(\sigma_{1}-1\right) \cdots\left(\sigma_{k}-1\right)$. On the other hand, using Lemma 3.1 recursively, we see that $s_{\sigma_{k}}\left(s_{\sigma_{k-1}}\left(\cdots\left(s_{\sigma_{1}}(x)\right) \cdots\right)\right)$ belongs to $\Gamma_{G}(k+1)$ for any $x \in G$. Hence

$$
s_{\sigma_{k}}\left(s_{\sigma_{k-1}}\left(\cdots\left(s_{\sigma_{1}}(x)\right) \cdots\right)\right)-1 \in \alpha_{k+1, G}\left(\mathcal{L}_{G}(k+1)\right) .
$$

By this lemma, in the following, we write the $k$-th Johnson homomorphism as

$$
\mu_{k}: Q^{k}(\operatorname{IA}(G)) \rightarrow \operatorname{Hom}_{\mathbf{Z}}\left(G^{\mathrm{ab}}, \alpha_{k+1, G}\left(\mathcal{L}_{G}(k+1)\right)\right) .
$$

Next, we consider a calculation of $\mu_{k+1}(a(\tau-1))$ for a given $a \in Q^{k}(\operatorname{IA}(G))$ and $\tau \in \operatorname{IA}(G)$. Let

$$
a=\sum_{\sigma_{1}, \ldots, \sigma_{k} \in \operatorname{IA}(G)} m_{\sigma_{1}, \ldots, \sigma_{k}}\left(\sigma_{1}-1\right) \cdots\left(\sigma_{k}-1\right)
$$

for $m_{\sigma_{1}, \ldots, \sigma_{k}} \in \mathbf{Z}$. Then for any $x \in G$, we have

$$
\begin{aligned}
\mu_{k+1}(a(\tau-1))(x) & =\sum_{\sigma_{1}, \ldots, \sigma_{k} \in \operatorname{IA}(G)} m_{\sigma_{1}, \ldots, \sigma_{k}} \mu_{k+1}\left(\left(\sigma_{1}-1\right) \cdots\left(\sigma_{k}-1\right)(\tau-1)\right)(x) \\
& \equiv \sum_{\sigma_{1}, \ldots, \sigma_{k} \in \operatorname{IA}(G)} m_{\sigma_{1}, \ldots, \sigma_{k}}\left\{s_{\tau}\left(s_{\sigma_{k}}\left(\cdots\left(s_{\sigma_{1}}(x)\right) \cdots\right)\right)-1\right\}
\end{aligned}
$$

modulo $\Delta^{k+3}(G)$. If we set $X:=s_{\sigma_{k}}\left(\cdots\left(s_{\sigma_{1}}(x)\right) \cdots\right) \in \Gamma_{G}(k+1)$, then

$$
\begin{aligned}
\mu_{k+1} & (a(\tau-1))(x) \\
& =\sum_{\sigma_{1}, \ldots, \sigma_{k} \in \operatorname{IA}(G)} m_{\sigma_{1}, \ldots, \sigma_{k}}\left\{X^{-1} X^{\tau}-1\right\} \\
& =\sum_{\sigma_{1}, \ldots, \sigma_{k} \in \operatorname{IA}(G)} m_{\sigma_{1}, \ldots, \sigma_{k}}\left\{\left(X^{-1}-1\right)\left(X^{\tau}-1\right)+\left(X^{-1}-1\right)+\left(X^{\tau}-1\right)\right\} \\
& \equiv \sum_{\sigma_{1}, \ldots, \sigma_{k} \in \operatorname{IA}(G)} m_{\sigma_{1}, \ldots, \sigma_{k}}\left\{\left(X^{\tau}-1\right)-(X-1)\right\} \\
& =\left\{\sum_{\sigma_{1}, \ldots, \sigma_{k} \in \operatorname{IA}(G)} m_{\sigma_{1}, \ldots, \sigma_{k}}(X-1)\right\}^{\tau}-\sum_{\sigma_{1}, \ldots, \sigma_{k} \in \operatorname{IA}(G)} m_{\sigma_{1}, \ldots, \sigma_{k}}(X-1) \\
& \equiv\left\{\mu_{k}(a)(x)\right\}^{\tau}-\mu_{k}(a)(x)
\end{aligned}
$$

modulo $\Delta^{k+3}(G)$. Hence we have

$$
\mu_{k+1}(a(\tau-1))(x)=\left\{\mu_{k}(a)(x)\right\}^{\tau}-\mu_{k}(a)(x) \in Q^{k+2}(\operatorname{IA}(G)) .
$$

This formula is sometimes convenient for a calculation of the image of $\mu_{k}$. 


\section{Free group case}

In this section, we mainly consider the case where $G=F_{n}$. For simplicity, we often omit the capital $F$ from the subscript $F_{n}$ if there is no confusion. For example, we write $\mathcal{L}_{n}, \mathcal{L}_{n}(k), \mathrm{IA}_{n}, \ldots$ for $\mathcal{L}_{F_{n}}, \mathcal{L}_{F_{n}}(k), \mathrm{IA}\left(F_{n}\right), \ldots$ respectively. Here, we study the structure of graded quotients $Q^{k}\left(\mathrm{IA}_{n}\right)$ as a $\operatorname{GL}(n, \mathbf{Z})$-module.

\subsection{Preliminary results for $G=F_{n}$}

In this subsection, we recall some well-known properties of the IA-automorphism group $\mathrm{IA}_{n}$, the graded Lie algebra $\mathcal{L}_{n}$ and the graded ring $\operatorname{gr}\left(\mathbf{Z}\left[F_{n}\right]\right)$. Let $H:=F_{n}^{\mathrm{ab}}$ be the abelianization of $F_{n}$. The natural homomorphism $\rho=\rho_{F_{n}}$ : Aut $F_{n} \rightarrow$ Aut $H$ induced from the abelianization of $F_{n} \rightarrow H$ is surjective. Throughout the paper, we identify Aut $H$ with the general linear group $\operatorname{GL}(n, \mathbf{Z})$ by fixing a basis of $H$ induced from the basis $x_{1}, \ldots, x_{n}$ of $F_{n}$. Namely, we have $\operatorname{GL}(n, \mathbf{Z}) \cong$ Aut $F_{n} / \mathrm{IA}_{n}$.

Magnus [13] showed that for any $n \geq 3, \mathrm{IA}_{n}$ is finitely generated by automorphisms

$$
K_{i j}: x_{t} \mapsto \begin{cases}x_{j}{ }^{-1} x_{i} x_{j} & t=i \\ x_{t} & t \neq i\end{cases}
$$

for distinct $1 \leq i, j \leq n$, and

$$
K_{i j l}: x_{t} \mapsto \begin{cases}x_{i}\left[x_{j}, x_{l}\right] & t=i, \\ x_{t} & t \neq i\end{cases}
$$

for distinct $1 \leq i, j, l \leq n$ and $j<l$. Recently, Cohen and Pakianathan [6; 7], Farb [8] and Kawazumi [12] independently showed

$$
\mathrm{IA}_{n}^{\mathrm{ab}} \cong H^{*} \otimes \mathbf{z} \Lambda^{2} H
$$

as a $\operatorname{GL}(n, \mathbf{Z})$-module. In particular, from their result, we see that $\mathrm{IA}_{n}^{\mathrm{ab}}$ is a free abelian group of rank $n^{2}(n-1) / 2$ with basis the coset classes of the Magnus generators $K_{i j}$ and $K_{i j l}$.

It is classically known due to Magnus that the graded Lie algebra $\mathcal{L}_{n}$ is isomorphic to the free Lie algebra generated by $H$ over $\mathbf{Z}$. (See Reutenauer [19], for example, for basic material concerning the free Lie algebra.) Each of the degree $k$ part $\mathcal{L}_{n}(k)$ of $\mathcal{L}_{n}$ is a free abelian group, which rank is given by Witt's formula

$$
\operatorname{rank}_{\mathbf{Z}}\left(\mathcal{L}_{n}(k)\right)=\frac{1}{k} \sum_{d \mid k} \mu(d) n^{k / d}
$$

where $\mu$ is the Möbius function. 
We next consider an embedding of the free Lie algebra $\mathcal{L}_{n}$ into the graded sum $\operatorname{gr}\left(\mathbf{Z}\left[F_{n}\right]\right)$. In general, it is known that the graded Lie algebra homomorphism $\alpha_{F_{n}}: \mathcal{L}_{n} \rightarrow \operatorname{gr}\left(\mathbf{Z}\left[F_{n}\right]\right)$ induced from $x \mapsto x-1$ for any $x \in F_{n}$ is a $\operatorname{GL}(n, \mathbf{Z})$-equivariant injective homomorphism, and that $\operatorname{gr}\left(\mathbf{Z}\left[F_{n}\right]\right)$ is naturally isomorphic to the universal enveloping algebra $\mathcal{U}\left(\mathcal{L}_{n}\right)$ of $\mathcal{L}_{n}$. (See [17, Theorem 6.2, Chapter VIII].) For simplicity, in the following, we identify $\mathcal{L}_{n}(k)$ with its image $\alpha_{k}\left(\mathcal{L}_{n}(k)\right)$ in $Q^{k}\left(F_{n}\right)$.

Here we observe a conjecture for the $\mathbf{Z}$-module structure of $Q^{k}\left(\mathrm{IA}_{n}\right)$. For a group $G$ such that each of the graded quotients $\mathcal{L}_{G}(k)$ is a free abelian group for $k \geq 1$, Sandling and Tahara [20] showed that as a $\mathbf{Z}$-module,

$$
Q^{k}(G) \cong \sum \bigotimes_{i=1}^{k} S^{a_{i}}\left(\mathcal{L}_{G}(i)\right)
$$

for each $k \geq 1$. Here $\sum$ runs over all nonnegative integers $a_{1}, \ldots, a_{k}$ such that

$$
\sum_{i=1}^{k} i a_{i}=k,
$$

and $S^{a}\left(\mathcal{L}_{G}(i)\right)$ means the symmetric tensor product of $\mathcal{L}_{G}(i)$ of degree $a$ such that $S^{0}\left(\mathcal{L}_{G}(i)\right)=\mathbf{Z}$.

On the other hand, it is conjectured by Andreadakis that the lower central series $\Gamma_{\mathrm{IA}_{n}}(k)$ coincides with the Johnson filtration $\mathcal{A}_{n}(k)$. He [1] showed that this is true for $n=2$. Since each of the graded quotient $\operatorname{gr}^{k}\left(\mathcal{A}_{n}\right):=\mathcal{A}_{n}(k) / \mathcal{A}_{n}(k+1)$ of the Johnson filtration $\mathcal{A}_{n}(k)$ is free abelian, the Andreadakis's conjecture let us conjecture:

Conjecture 4.1 For any $k \geq 1$,

$$
Q^{k}\left(\mathrm{IA}_{n}\right) \cong \sum \bigotimes_{i=1}^{k} S^{a_{i}}\left(\mathcal{L}_{\mathrm{IA}_{n}}(i)\right)
$$

as a $\mathbf{Z}$-module. Here the sum runs over all nonnegative integers $a_{1}, \ldots, a_{k}$ such that $\sum_{i=1}^{k} i a_{i}=k$.

To study $Q^{k}\left(\mathrm{IA}_{n}\right)$, first, we consider the surjective homomorphism $\pi_{k}: Q^{k}\left(\mathrm{IA}_{n}\right) \rightarrow$ $Q^{k}\left(\mathrm{IA}_{n}^{\mathrm{ab}}\right)$ induced from the abelianization of $\mathrm{IA}_{n}$ for $k \geq 1$. We remark that each of $\pi_{k}$ is an $\operatorname{GL}(n, \mathbf{Z})$-equivariant surjective homomorphism, and that $Q^{k}\left(\mathrm{IA}_{n}^{\mathrm{ab}}\right) \cong S^{k}\left(\mathrm{IA}_{n}^{\mathrm{ab}}\right)$ since $\mathrm{IA}_{n}^{\mathrm{ab}}$ is free abelian as mentioned before. For $k=1, \pi_{k}: Q^{1}\left(\mathrm{IA}_{n}\right) \rightarrow Q^{1}\left(\mathrm{IA}_{n}^{\mathrm{ab}}\right)$ is an isomorphism, and $Q^{1}\left(\mathrm{IA}_{n}\right) \cong \mathrm{IA}_{n}^{\mathrm{ab}}=H^{*} \otimes_{\mathrm{z}} \Lambda^{2} H$. In general, however, $\pi_{k}$ is not injective for $k \geq 2$, and seems to have a large kernel from the conjecture above. In this paper, to investigate the $\operatorname{GL}(n, \mathbf{Z})$-module structure of $\operatorname{Ker}\left(\pi_{k}\right)$, we use the Johnson homomorphism $\mu_{k}$. 


\subsection{The image of $\mu_{k} \mid \operatorname{Ker}\left(\pi_{k}\right)$}

Here we study the image of the Johnson homomorphism

$$
\mu_{k}: Q^{k}\left(\mathrm{IA}_{n}\right) \rightarrow H^{*} \otimes_{\mathbf{z}} \mathcal{L}_{n}(k+1) \subset H^{*} \otimes_{\mathbf{z}} Q^{k+1}\left(F_{n}\right)
$$

restricted to the kernel of $\pi_{k}$ for a sufficiently large $n$. Note that $H^{*} \otimes_{\mathbf{Z}} \mathcal{L}_{n}(k+1)=$ $H^{*} \otimes_{\mathbf{Z}} \alpha_{k+1}\left(\mathcal{L}_{n}(k+1)\right)$ is generated by elements

$$
x_{i}^{*} \otimes\left(\left[x_{i_{1}}, \ldots, x_{i_{k+1}}\right]-1\right), \quad 1 \leq i, i_{j} \leq n
$$

as a $\mathbf{Z}$-module. First we consider the case where $k \geq 3$.

Proposition 4.2 For any $k \geq 3$ and $n \geq k+2$, the homomorphism

$$
\left.\mu_{k}\right|_{\operatorname{Ker}\left(\pi_{k}\right)}: \operatorname{Ker}\left(\pi_{k}\right) \rightarrow H^{*} \otimes_{\mathbf{Z}} \mathcal{L}_{n}(k+1)
$$

is surjective.

Proof For any $x_{i}^{*} \otimes\left(\left[x_{i_{1}}, \ldots, x_{i_{k+1}}\right]-1\right)$, since $n \geq k+2$, there exists some $1 \leq j \leq n$ such that $j \neq i_{1}, \ldots, i_{k+1}$.

Case 1 The case where $i_{k+1} \neq i$. Set

$$
a:= \begin{cases}\left(K_{i j i_{k+1}}-1\right)\left(K_{j i_{k}}-1\right) \cdots\left(K_{j i_{3}}-1\right)\left(K_{j i_{1} i_{2}}-1\right) & \text { if } j \neq i, \\ \left(K_{j i_{k+1}}-1\right)\left(K_{j i_{k}}-1\right) \cdots\left(K_{j i_{3}}-1\right)\left(K_{j i_{1} i_{2}}-1\right) & \text { if } j=i .\end{cases}
$$

Then we have $\mu_{k}(a)=x_{i}^{*} \otimes\left(\left[x_{i_{1}}, \ldots, x_{i_{k+1}}\right]-1\right)$. On the other hand, if we set

$$
b:= \begin{cases}\left(K_{j i_{1} i_{2}}-1\right)\left(K_{j i_{3}}-1\right) \cdots\left(K_{j i_{k}}-1\right)\left(K_{i j i_{k+1}}-1\right) & \text { if } j \neq i, \\ \left(K_{j i_{1} i_{2}}-1\right)\left(K_{j i_{3}}-1\right) \cdots\left(K_{j i_{k}}-1\right)\left(K_{j i_{k+1}}-1\right) & \text { if } j=i,\end{cases}
$$

then $\mu_{k}(b)=0$. Hence we obtain $\mu_{k}(a-b)=x_{i}^{*} \otimes\left(\left[x_{i_{1}}, \ldots, x_{i_{k+1}}\right]-1\right)$ for $a-b \in \operatorname{Ker}\left(\pi_{k}\right)$.

Case 2 The case where $i_{k+1}=i$. Set

$$
a^{\prime}:=\left(K_{i j}^{-1}-1\right)\left(K_{j i_{k}}-1\right) \cdots\left(K_{j i_{3}}-1\right)\left(K_{j i_{1} i_{2}}-1\right) .
$$

Then $\mu_{k}\left(a^{\prime}\right)=x_{i}^{*} \otimes\left(\left[x_{i_{1}}, \ldots, x_{i_{k+1}}\right]-1\right)$. On the other hand, if we set

$$
b^{\prime}:=\left(K_{j i_{1} i_{2}}-1\right)\left(K_{j i_{3}}-1\right) \cdots\left(K_{j i_{k}}-1\right)\left(K_{i j}^{-1}-1\right),
$$

$\mu_{k}\left(b^{\prime}\right)=0$. Hence we obtain $\mu_{k}\left(a^{\prime}-b^{\prime}\right)=x_{i}^{*} \otimes\left(\left[x_{i_{1}}, \ldots, x_{i_{k+1}}\right]-1\right)$ for $a^{\prime}-b^{\prime} \in$ $\operatorname{Ker}\left(\pi_{k}\right)$. This completes the proof of Proposition 4.2. 
It seems to difficult to show above for $2 \leq n \leq k+2$ since we can not take $1 \leq j \leq n$ such that $j \neq i_{1}, \ldots, i_{k+1}$ in general.

As a corollary to Proposition 4.2, we see the surjectivity of $\mu_{k}$ of $\mathbf{Z}[\operatorname{IA}(G)]$ for the case where $G$ is a certain quotient group of $F_{n}$. Let $C$ be a characteristic subgroup of $F_{n}$ such that $C \subset \Gamma_{n}(2)$, and set $G:=F_{n} / C$. Then we have a natural isomorphism $G^{\mathrm{ab}} \cong H$. The natural projection $\phi: F_{n} \rightarrow G$ induces homomorphisms $Q^{k}\left(F_{n}\right) \rightarrow Q^{k}(G)$, also denoted by $\phi$. Since $C$ is characteristic, $\phi: F_{n} \rightarrow G$ induces a homomorphism $\bar{\phi}$ : Aut $F_{n} \rightarrow \operatorname{Aut}(G)$. Clearly, $\bar{\phi}\left(\operatorname{IA}_{n}\right) \subset \operatorname{IA}(G)$. Furthermore, $\bar{\phi}$ naturally induces homomorphisms $Q^{k}\left(\mathrm{IA}_{n}\right) \rightarrow Q^{k}(\mathrm{IA}(G))$ which is also denoted by $\bar{\phi}$.

Corollary 4.3 With the notation above, for any $k \geq 3$ and $n \geq k+2$, the homomorphism $\mu_{k}: \operatorname{Ker}\left(\pi_{k, \mathrm{IA}(G)}\right) \rightarrow H^{*} \otimes_{\mathbf{Z}} \alpha_{k+1}\left(\mathcal{L}_{G}(k+1)\right)$ is surjective.

Proof It is clear from a commutative diagram

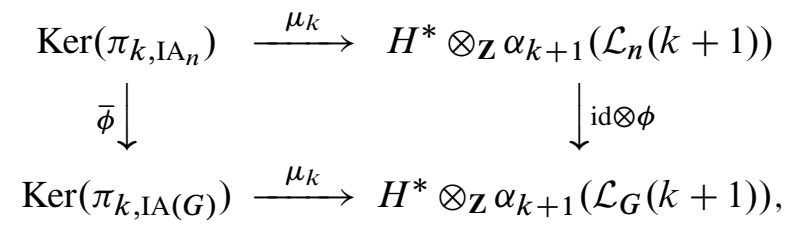

where the first row and id $\otimes \phi$ are surjective.

For example, if $G$ is a free metabelian group $G=F_{n} /\left[\Gamma_{n}(2), \Gamma_{n}(2)\right]$, then the Johnson homomorphism $\mu_{k}: \operatorname{Ker}\left(\pi_{k, \mathrm{IA}(G)}\right) \rightarrow H^{*} \otimes_{\mathbf{Z}} \alpha_{k+1}\left(\mathcal{L}_{G}(k+1)\right)$ is surjective for any $k \geq 3$ and $n \geq k+2$. In Section 5, we show that we can improve the condition $k \geq 3$ and $n \geq k+2$ above for $G=F_{n} /\left[\Gamma_{n}(2), \Gamma_{n}(2)\right]$.

By Proposition 4.2 and Corollary 4.3, we have:

Theorem 4.4 Let $C$ and $G$ be as above. For $k \geq 3$ and $n \geq k+2$, an $\operatorname{Aut}(G) / \operatorname{IA}(G)-$ equivariant homomorphism

$$
\mu_{k} \oplus \pi_{k}: Q^{k}(\operatorname{IA}(G)) \rightarrow\left(H^{*} \otimes_{\mathbf{z}} \alpha_{k+1, G}\left(\mathcal{L}_{G}(k+1)\right)\right) \bigoplus Q^{k}\left(\operatorname{IA}(G)^{\mathrm{ab}}\right)
$$

defined by $\sigma \mapsto\left(\mu_{k}(\sigma), \pi_{k}(\sigma)\right)$ is surjective.

In particular, for $C=\{1\}$, and hence $G=F_{n}$, we have a $\operatorname{GL}(n, \mathbf{Z})$-equivariant surjective homomorphism

$$
\mu_{k} \oplus \pi_{k}: Q^{k}\left(\mathrm{IA}_{n}\right) \rightarrow\left(H^{*} \otimes_{\mathrm{z}} \mathcal{L}_{n}(k+1)\right) \oplus S^{k}\left(\mathrm{IA}_{n}^{\mathrm{ab}}\right)
$$

for $k \geq 3$ and $n \geq k+2$. 
Finally, we consider the case where $k=2$. Observing a split exact sequence (1), we see that $\operatorname{Ker}\left(\pi_{2}\right)=\alpha_{2, \operatorname{IA}(G)}\left(\mathcal{L}_{\mathrm{IA}(G)}(2)\right)$. Hence, from the compatibility (2), we see that $\operatorname{Im}\left(\left.\mu_{2}\right|_{\operatorname{Ker}\left(\pi_{2}\right)}\right)=\alpha_{3, F_{n}}^{*}\left(\operatorname{Im}\left(\tau_{2}^{\prime}\right)\right)$. In [21], we showed that for any $n \geq 2, \operatorname{Im}\left(\tau_{2}^{\prime}\right)$, which is equal to $\operatorname{Im}\left(\tau_{2}\right)$, satisfies an exact sequence

$$
0 \rightarrow \operatorname{Im}\left(\tau_{2}^{\prime}\right) \stackrel{\tau_{2}^{\prime}}{\rightarrow} H^{*} \otimes_{\mathbf{z}} \mathcal{L}_{n}(3) \rightarrow S^{2} H \rightarrow 0
$$

of $\operatorname{GL}(n, \mathbf{Z})$-modules. Hence we see that:

Proposition 4.5 For $n \geq 2, \operatorname{Im}\left(\left.\mu_{2}\right|_{\operatorname{Ker}\left(\pi_{2}\right)}\right)$ is a $\operatorname{GL}(n, \mathbf{Z})$-equivariant proper submodule of $H^{*} \otimes \mathbf{Z} \alpha_{3}\left(\mathcal{L}_{n}(3)\right)$, which rank is given by

$$
\frac{1}{6} n(n+1)\left(2 n^{2}-2 n-3\right)
$$

Here we remark that $\mu_{2}$ is surjective.

Lemma 4.6 For any $n \geq 2$, the map $\mu_{2}: Q^{2}\left(\mathrm{IA}_{n}\right) \rightarrow H^{*} \otimes_{\mathrm{z}} \mathcal{L}_{n}(3)$ is surjective.

Proof Take an element $x_{i}^{*} \otimes\left(\left[x_{i_{1}}, x_{i_{2}}, x_{i_{3}}\right]-1\right)$. We may assume $i_{1} \neq i_{2}$. If $i_{j} \neq i$ for $1 \leq j \leq 3$, we see that

$$
\mu_{2}\left(\left(K_{i i_{3}}-1\right)\left(K_{i i_{1} i_{2}}-1\right)\right)=x_{i}^{*} \otimes\left(\left[x_{i_{1}}, x_{i_{2}}, x_{i_{3}}\right]-1\right) .
$$

If $i_{3}=i$ and $i_{1}, i_{2} \neq i$, then

$$
\mu_{2}\left(\left(K_{i i_{1}}^{-1}-1\right)\left(K_{i_{1} i_{2}}-1\right)\right)=x_{i}^{*} \otimes\left(\left[x_{i_{1}}, x_{i_{2}}, x_{i}\right]-1\right) .
$$

If $i_{1}=i$ and $i_{2}, i_{3} \neq i$, then

$$
\mu_{2}\left(\left(K_{i i_{3}}-1\right)\left(K_{i i_{2}}-1\right)\right)=x_{i}^{*} \otimes\left(\left[x_{i}, x_{i_{2}}, x_{i_{3}}\right]-1\right) .
$$

If $i_{2}=i$ and $i_{1}, i_{3} \neq i$, then

$$
\mu_{2}\left(\left(K_{i i_{3}}-1\right)\left(K_{i i_{1}}^{-1}-1\right)\right)=x_{i}^{*} \otimes\left(\left[x_{i_{1}}, x_{i}, x_{i_{3}}\right]-1\right) .
$$

If $i_{1}=i_{3}=i$, then

$$
\mu_{2}\left(\left(K_{i i_{2}}^{-1}-1\right)\left(K_{i_{2} i}^{-1}-1\right)\right)=x_{i}^{*} \otimes\left(\left[x_{i}, x_{i_{2}}, x_{i}\right]-1\right) .
$$

If $i_{2}=i_{3}=i$, then

$$
\mu_{2}\left(\left(K_{i i_{1}}^{-1}-1\right)\left(K_{i_{1} i}^{-1}-1\right)\right)=x_{i}^{*} \otimes\left(\left[x_{i_{1}}, x_{i}, x_{i}\right]-1\right) .
$$

Hence the generators of $H^{*} \otimes_{\mathbf{Z}} \mathcal{L}_{n}(3)$ are contained in the image of $\mu_{2}$. 


\section{Free metabelian case}

In this section, we mainly consider the case where $G=F_{n}^{M}:=F_{n} /\left[\Gamma_{n}(2), \Gamma_{n}(2)\right]$. For simplicity, we often omit the capital $F$ from the subscript $F_{n}^{M}$ if there is no confusion. For example, we write $\mathcal{L}_{n}^{M}, \mathcal{L}_{n}^{M}(k), \mathrm{IA}_{n}^{M}, \ldots$, for $\mathcal{L}_{F_{n}^{M}}, \mathcal{L}_{F_{n}^{M}}(k)$, $\mathrm{IA}\left(F_{n}^{M}\right), \ldots$, respectively. Here, we study the structure of graded quotients $Q^{k}\left(\mathrm{IA}_{n}^{M}\right)$ as a $\operatorname{GL}(n, \mathbf{Z})$-module.

\subsection{Preliminary results for $G=F_{n}^{M}$}

In this subsection, we recall some properties of the IA-automorphism group $\mathrm{IA}_{n}^{M}$ and the graded Lie algebras $\mathcal{L}_{n}^{M}$.

To begin with, we have $\left(F_{n}^{M}\right)^{\mathrm{ab}}=H$, and hence $\operatorname{Aut}\left(F_{n}^{M}\right)^{\mathrm{ab}}=\operatorname{Aut}(H)=\operatorname{GL}(n, \mathbf{Z})$. Since the surjective map $\rho_{F_{n}}$ : Aut $F_{n} \rightarrow \operatorname{GL}(n, \mathbf{Z})$ factors through Aut $F_{n}^{M}$, a map $\rho_{F_{n}^{M}}$ : Aut $F_{n}^{M} \rightarrow \mathrm{GL}(n, \mathbf{Z})$ is also surjective. So we can identify $\operatorname{Aut} F_{n}^{M} / \operatorname{IA}\left(F_{n}^{M}\right)$ with $\operatorname{GL}(n, \mathbf{Z})$.

Let $v_{n}$ : Aut $F_{n} \rightarrow$ Aut $F_{n}^{M}$ be the natural homomorphism induced from the action of Aut $F_{n}$ on $F_{n}^{M}$. Restricting $v_{n}$ to $\mathrm{IA}_{n}$ gives a homomorphism $\left.v_{n}\right|_{\mathrm{IA}_{n}}: \mathrm{IA}_{n} \rightarrow \mathrm{IA}_{n}^{M}$. Bachmuth and Mochizuki [4] showed that $\left.v_{n}\right|_{\mathrm{IA}_{n}}$ is surjective for $n \geq 4$. They also showed that $\left.v_{3}\right|_{\mathrm{IA}_{3}}$ is not surjective and $\mathrm{IA}_{3}^{M}$ is not finitely generated [3]. Hence $\mathrm{IA}_{n}^{M}$ is finitely generated for $n \geq 4$ by the (coset classes of) Magnus generators $K_{i j}$ and $K_{i j l}$. We remark that since $\operatorname{Ker}\left(\left.v_{n}\right|_{\mathrm{IA}_{n}}\right)$ is contained in $\mathcal{A}_{n}(3)$, we have isomorphisms

$$
\left(\mathrm{IA}_{n}^{M}\right)^{\mathrm{ab}} \cong \mathrm{IA}_{n}^{\mathrm{ab}} \cong H^{*} \otimes \mathrm{z} \Lambda^{2} H
$$

as a $\operatorname{GL}(n, \mathbf{Z})$-module.

The associated Lie algebra $\mathcal{L}_{n}^{M}=\bigoplus_{k \geq 1} \mathcal{L}_{n}^{M}(k)$ is called the free metabelian Lie algebra generated by $H$ or the Chen Lie algebra. It is also classically known due to Chen [5] that each $\mathcal{L}_{n}^{M}(k)$ is a $\operatorname{GL}(n, \mathbf{Z})$-equivariant free abelian group of rank

$$
\operatorname{rank}_{\mathbf{Z}}\left(\mathcal{L}_{n}^{M}(k)\right):=(k-1)\left(\begin{array}{c}
n+k-2 \\
k
\end{array}\right) .
$$

We remark that $\mathcal{L}_{n}(k)=\mathcal{L}_{n}^{M}(k)$ for $1 \leq k \leq 3$.

By the same argument as in Section 4.1, for each $k \geq 2$, we can detect $S^{k}\left(\left(\operatorname{IA}_{n}^{M}\right)^{\mathrm{ab}}\right)$ in $Q^{k}\left(\mathrm{IA}_{n}^{M}\right)$ by the $\operatorname{GL}(n, \mathbf{Z})$-equivariant surjective homomorphism $\pi_{k}^{M}: Q^{k}\left(\mathrm{IA}_{n}^{M}\right) \rightarrow$ $Q^{k}\left(\left(\mathrm{IA}_{n}^{M}\right)^{\mathrm{ab}}\right)$ induced from the abelianization of $\mathrm{IA}_{n}^{M}$. In order to investigate the $\operatorname{GL}(n, \mathbf{Z})$-module structure of $\operatorname{Ker}\left(\pi_{k}^{M}\right)$, we use the Johnson homomorphism $\mu_{k}$. 


\subsection{The image of $\left.\mu_{k}\right|_{\operatorname{Ker}\left(\pi_{k}^{M}\right)}$}

Here we study the image of the Johnson homomorphism

$$
\mu_{k}: Q^{k}\left(\mathrm{IA}_{n}^{M}\right) \rightarrow H^{*} \otimes_{\mathrm{Z}} \alpha_{k+1}\left(\mathcal{L}_{n}^{M}(k+1)\right)
$$

restricted to the kernel of $\pi_{k}^{M}$ for $n \geq 4$. First, in order to get a reasonable generators of $\mathcal{L}_{n}^{M}(k+1)$, we consider some lemmas. Let $\mathfrak{S}_{l}$ be the symmetric group of degree $l$. Then we have:

Lemma 5.1 Let $l \geq 2$ and $n \geq 2$. For any element $\left[x_{i_{1}}, x_{i_{2}}, x_{j_{1}}, \ldots, x_{j_{l}}\right] \in \mathcal{L}_{n}^{M}(l+2)$ and any $\lambda \in \mathfrak{S}_{l}$,

$$
\left[x_{i_{1}}, x_{i_{2}}, x_{j_{1}}, \ldots, x_{j_{l}}\right]=\left[x_{i_{1}}, x_{i_{2}}, x_{j_{\lambda(1)}} \ldots, x_{j_{\lambda(l)}}\right] .
$$

Proof Since $\mathfrak{S}_{l}$ is generated by transpositions $(m m+1)$ for $1 \leq m \leq l-1$, it suffices to prove the lemma for each $\lambda=(m m+1)$. Now we have

$$
\begin{aligned}
& \left.\left[\left[\left[x_{i_{1}}, x_{i_{2}}, x_{j_{1}}, \ldots, x_{j_{m-1}}\right], x_{j_{m}}\right], x_{j_{m+1}}\right]\right] \\
& \quad=-\left[\left[x_{j_{m}}, x_{j_{m+1}}\right],\left[x_{i_{1}}, x_{i_{2}}, x_{j_{1}}, \ldots, x_{j_{m-1}}\right]\right] \\
& \quad-\left[\left[x_{j_{m+1}},\left[x_{i_{1}}, x_{i_{2}}, x_{j_{1}}, \ldots, x_{j_{m-1}}\right]\right], x_{j_{m}}\right] \\
& \left.\quad\left[\left[x_{i_{1}}, x_{i_{2}}, x_{j_{1}}, \ldots, x_{j_{m-1}}\right], x_{j_{m+1}}\right], x_{j_{m}}\right]
\end{aligned}
$$

in $\mathcal{L}_{n}^{M}(m+3)$ by the Jacobi identity. Hence,

$$
\begin{aligned}
{\left[x_{i_{1}}, x_{i_{2}}, x_{j_{1}}, \ldots, x_{j_{l}}\right] } & =\left[x_{i_{1}}, x_{i_{2}}, x_{j_{1}}, \ldots, x_{j_{m-1}}, x_{j_{m+1}}, x_{j_{m}}, \ldots, x_{j_{l}}\right] \\
& =\left[x_{i_{1}}, x_{i_{2}}, x_{j_{\lambda(1)}} \ldots, x_{j_{\lambda(l)}}\right] .
\end{aligned}
$$

in $\mathcal{L}_{n}^{M}(l+2)$.

Similarly to $H^{*} \otimes_{\mathbf{Z}} \alpha_{k+1}\left(\mathcal{L}_{n}(k+1)\right)$, the $\mathbf{Z}$-module $H^{*} \otimes_{\mathbf{Z}} \alpha_{k+1}\left(\mathcal{L}_{n}^{M}(k+1)\right)$ is generated by elements

$$
x_{i}^{*} \otimes\left(\left[x_{i_{1}}, \ldots, x_{i_{k+1}}\right]-1\right), \quad 1 \leq i, i_{j} \leq n .
$$

On the other hand, using Lemma 5.1, elements $\left[x_{i_{1}}, x_{i_{2}}, \ldots, x_{i_{k+1}}\right] \in \mathcal{L}_{n}^{M}(k+1)$ is rewritten as

$$
\left[x_{i_{1}}, x_{i_{2}}, x_{i_{3}}, \ldots, x_{i_{l-1}}, x_{i}, x_{i}, \ldots, x_{i}\right]
$$

in $\mathcal{L}_{n}^{M}(k+1)$ for some $l, 3 \leq l \leq k+2$ such that $i_{3}, i_{4}, \ldots, i_{l-1} \neq i$. Hence $H^{*} \otimes_{\mathbf{Z}} \alpha_{k+1}\left(\mathcal{L}_{n}^{M}(k+1)\right)$ is generated by elements

$$
x_{i}^{*} \otimes\left(\left[x_{i_{1}}, x_{i_{2}}, x_{i_{3}}, \ldots, x_{i_{l-1}}, x_{i}, x_{i}, \ldots, x_{i}\right]-1\right)
$$


for some $l, 3 \leq l \leq k+2$ such that $i_{3}, \ldots, i_{l-1} \neq i$. Furthermore, without loss of generality, we may assume $i_{2} \neq i$ in the generators above.

Proposition 5.2 For any $k \geq 2$ and $n \geq 4$, the homomorphism

$$
\left.\mu_{k}\right|_{\operatorname{Ker}\left(\pi_{k}^{M}\right)}: \operatorname{Ker}\left(\pi_{k}^{M}\right) \rightarrow H^{*} \otimes_{\mathbf{z}} \oslash_{k+1}\left(\mathcal{L}_{n}^{M}(k+1)\right)
$$

is surjective.

Proof Take a generator

$$
x_{i}^{*} \otimes\left(\left[x_{i_{1}}, x_{i_{2}}, x_{i_{3}}, \ldots, x_{i_{l-1}}, x_{i}, x_{i}, \ldots, x_{i}\right]-1\right)
$$

of $H^{*} \otimes_{\mathbf{Z}} \alpha_{k+1}\left(\mathcal{L}_{n}^{M}(k+1)\right)$ for some $l, 3 \leq l \leq k+2$ such that $i_{2}, \ldots, i_{l-1} \neq i$ as mentioned above. Since $n \geq 4$, there exists some $1 \leq j \leq n$ such that $j \neq i, i_{1}, i_{2}$. First, consider an element

$$
a:=\left(K_{i j}^{-1}-1\right)\left(K_{j i}-1\right) \cdots\left(K_{j i}-1\right) \in \Delta^{k-l+2}\left(\mathrm{IA}_{n}^{M}\right),
$$

where $\left(K_{j i}-1\right)$ appears $k-l+1$ times in the product. Then we see

$$
\mu_{k-l+3}(a)=x_{i}^{*} \otimes\left(\left[x_{j}, x_{i}, \ldots, x_{i}\right]-1\right),
$$

where $x_{i}$ appears $k-l+2$ times among the component.

Next, set

$$
\begin{aligned}
& b:= \begin{cases}K_{j i i_{l-1}-1} \text { if } j \neq i_{l-1}, \\
K_{j i}^{-1}-1 & \text { if } j=i_{l-1},\end{cases} \\
& c:=\left(K_{\left.i i_{l-2}-1\right)\left(K_{i i_{l-3}}-1\right) \cdots\left(K_{i i_{3}}-1\right) \in \Delta^{l-4}\left(\mathrm{IA}_{n}^{M}\right),}\right. \\
& d:= \begin{cases}K_{i i_{1} i_{2}}-1 & \text { if } i \neq i_{1}, \\
K_{i i_{2}}-1 & \text { if } i=i_{1} .\end{cases}
\end{aligned}
$$

Then we have

$$
\mu_{k}(a b c d)=x_{i}^{*} \otimes\left(\left[x_{i_{1}}, x_{i_{2}}, x_{i_{3}}, \ldots, x_{i_{l-1}}, x_{i}, x_{i}, \ldots, x_{i}\right]-1\right) .
$$

On the other hand, $\mu_{k}(\mathrm{dbac})=0$. Hence we have

$$
\mu_{k}(a b c d-d b a c)=x_{i}^{*} \otimes\left(\left[x_{i_{1}}, x_{i_{2}}, x_{i_{3}}, \ldots, x_{i_{l-1}}, x_{i}, x_{i}, \ldots, x_{i}\right]-1\right) .
$$

Therefore since $a b c d-d b a c \in \operatorname{Ker}\left(\pi_{k}^{M}\right)$, we conclude that $\left.\mu_{k}\right|_{\operatorname{Ker}\left(\pi_{k}^{M}\right)}$ is surjective. This completes the proof of Proposition 5.2. 
Then we have:

Theorem 5.3 For $k \geq 2$ and $n \geq 4$, a $\operatorname{GL}(n, \mathbf{Z})$-equivariant homomorphism

$$
\mu_{k} \oplus \pi_{k}: Q^{k}\left(\mathrm{IA}_{n}^{M}\right) \rightarrow\left(H^{*} \otimes_{\mathrm{z}} \alpha_{k+1}\left(\mathcal{L}_{n}^{M}(k+1)\right)\right) \bigoplus S^{k}\left(\left(\mathrm{IA}_{n}^{M}\right)^{\mathrm{ab}}\right)
$$

defined by $\sigma \mapsto\left(\mu_{k}(\sigma), \pi_{k}(\sigma)\right)$ is surjective.

Acknowledgements The author would like to thank the referee for correcting a number of typos and grammatical errors of the original version of this article. Furthermore, he also thanks to the referee for pointing out a mathematical error in the proof of Lemma 3.8 and for helpful comments for it.

This research is supported by a JSPS Research Fellowship for Young Scientists and the Global COE program at Kyoto University.

\section{References}

[1] S Andreadakis, On the automorphisms of free groups and free nilpotent groups, Proc. London Math. Soc. 15 (1965) 239-268 MR0188307

[2] S Bachmuth, Induced automorphisms of free groups and free metabelian groups, Trans. Amer. Math. Soc. 122 (1966) 1-17 MR0190212

[3] S Bachmuth, H Y Mochizuki, The nonfinite generation of $\operatorname{Aut}(G), G$ free metabelian of rank 3, Trans. Amer. Math. Soc. 270 (1982) 693-700 MR645339

[4] S Bachmuth, H Y Mochizuki, $\operatorname{Aut}(F) \rightarrow \operatorname{Aut}\left(F / F^{\prime \prime}\right)$ is surjective for free group $F$ of rank $\geq 4$, Trans. Amer. Math. Soc. 292 (1985) 81-101 MR805954

[5] K-T Chen, Integration in free groups, Ann. of Math. 54 (1951) 147-162 MR0042414

[6] F Cohen, J Pakianathan, On automorphism groups of free groups, and their nilpotent quotients, in preparation

[7] F Cohen, J Pakianathan, On subgroups of the automorphism group of a free group and associated graded Lie algebras, in preparation

[8] B Farb, Automorphisms of $F_{n}$ which act trivially on homology, in preparation

[9] R Hain, Infinitesimal presentations of the Torelli groups, J. Amer. Math. Soc. 10 (1997) 597-651 MR1431828

[10] D Johnson, An abelian quotient of the mapping class group $\mathcal{I}_{g}$, Math. Ann. 249 (1980) 225-242 MR579103

[11] D Johnson, The structure of the Torelli group-III: The abelianization of $\mathcal{I}$, Topology 24 (1985) 127-144 MR793179 
[12] N Kawazumi, Cohomological aspects of Magnus expansions arXiv: math.GT/0505497

[13] W Magnus, Über n-dimensionale Gittertransformationen, Acta Math. 64 (1935) 353367 MR1555401

[14] S Morita, Abelian quotients of subgroups of the mapping class group of surfaces, Duke Math. J. 70 (1993) 699-726 MR1224104

[15] S Morita, Structure of the mapping class groups of surfaces: a survey and a prospect, from: "Proceedings of the Kirbyfest (Berkeley, CA, 1998)", (J Hass, M Scharlemann, editors), Geom. Topol. Monogr. 2, Geom. Topol. Publ., Coventry (1999) 349-406 MR1734418

[16] S Morita, Cohomological structure of the mapping class group and beyond, from: "Problems on mapping class groups and related topics", (B Farb, editor), Proc. Sympos. Pure Math. 74, Amer. Math. Soc. (2006) 329-354 MR2264550

[17] I B S Passi, Group rings and their augmentation ideals, Lecture Notes in Math. 715, Springer, Berlin (1979) MR537126

[18] A Pettet, The Johnson homomorphism and the second cohomology of $\mathrm{IA}_{n}$, Algebr. Geom. Topol. 5 (2005) 725-740 MR2153110

[19] C Reutenauer, Free Lie algebras, London Math. Soc. Monogr. 7, Oxford Science Publ., The Clarendon Press, Oxford Univ. Press, New York (1993) MR1231799

[20] R Sandling, K-I Tahara, Augmentation quotients of group rings and symmetric powers, Math. Proc. Cambridge Philos. Soc. 85 (1979) 247-252 MR516083

[21] T Satoh, New obstructions for the surjectivity of the Johnson homomorphism of the automorphism group of a free group, J. London Math. Soc. 74 (2006) 341-360 MR2269583

[22] T Satoh, The cokernel of the Johnson homomorphisms of the automorphism group of a free metabelian group, Trans. Amer. Math. Soc. 361 (2009) 2085-2107 MR2465830

[23] T Satoh, On the lower central series of the IA-automorphism group of a free group, J. Pure Appl. Algebra 216 (2012) 709-717 MR2864772

Department of Mathematics, Faculty of Science, Tokyo University of Science 1-3 Kagurazaka, Shinjuku-ku, Tokyo 162-8601, Japan

takao@rs.tus.ac.jp

Received: 4 April $2011 \quad$ Revised: 22 March 2012 